\title{
Physical properties of a sunspot chromosphere with umbral flashes ${ }^{\star}$
}

\author{
J. de la Cruz Rodríguez ${ }^{1}$, L. Rouppe van der Voort ${ }^{2}$, H. Socas-Navarro ${ }^{3}$, and M. van Noort ${ }^{4}$ \\ ${ }^{1}$ Department of Physics and Astronomy, Uppsala University, Box 516, 75120 Uppsala, Sweden \\ e-mail: jaime.cruz@physics.uu.se \\ 2 Institute of Theoretical Astrophysics, University of Oslo, PO Box 1029 Blindern, 0315 Oslo, Norway \\ 3 Instituto de Astrofísica de Canarias, Avda vía Láctea S/N, 38205 La Laguna, Tenerife, Spain \\ ${ }^{4}$ Max-Planck Institute for Solar System Research, Max-Planck Straße 1, 37412 Katlenburg-Lindau, Germany
}

Received 2 April 2013 / Accepted 21 June 2013

\begin{abstract}
We present new high-resolution spectro-polarimetric Ca II $\lambda 8542$ observations of umbral flashes in sunspots. At nearly 0 '. 18 , and spanning about one hour of continuous observation, this is the most detailed dataset published thus far. Our study involves both LTE and non-LTE inversions (but includes also a weak field analysis as a sanity check) to quantify temperatures, mass flows and the full magnetic field vector geometry. We confirm earlier reports that UFs have very fine structure with hot and cool material intermixed at sub-arcsecond scales. The shock front is roughly $1000 \mathrm{~K}$ hotter than the surrounding material. We do not observe significant fluctuations of the field in the umbra. In the penumbra, however, the passage of the running penumbral waves alter the magnetic field strength by some $200 \mathrm{G}$ (peak-to-peak amplitude) but it does not change the field orientation (at least not significantly within our sensitivity of a few degrees). From a fast Fourier transform analysis, we find a trend of decreasing power at high temporal frequencies at those locations with more horizontal magnetic fields, for the line-of-sight velocity and magnetic field strength. In the outer penumbra we find an absence of high frequency power while there is increasingly more power at high frequencies towards the umbra.
\end{abstract}

Key words. Sun: chromosphere - sunspots - Sun: magnetic topology - radiative transfer - polarization - Sun: infrared

\section{Introduction}

Umbral flashes (UFs) are sudden brightenings observed in the chromospheric core of the Ca II lines. They tend to appear with a periodicity of roughly 3 min (Beckers \& Tallant 1969; Wittmann 1969) and it is now reasonably well established that they are the chromospheric counterpart of the photospheric oscillation (Kneer et al. 1981; Thomas 1984). The waves, propagating from the underlying photosphere, steepen and become non-linear as the atmospheric density drops by several orders of magnitude. Eventually, the waves develop into shocks which are observable as blue-shifted emission reversals in the Ca II line cores. A semi-empirical study by Felipe et al. (2011) allowed to reproduce multi-line sunspot observations using a 3D non-linear MHD code (Felipe et al. 2010). A more theoretical study by Bard \& Carlsson (2010) analyzed which modes contribute to the formation of UFs and investigated the role of the coronal radiation in the strength of these shock events.

The development of spectro-polarimetry has paved the way for new advances in our understanding of UFs, mostly aided by the characteristic nearly anti-symmetric shape of the Stokes $V$ profile. The first polarimetric observations of UFs (Socas-Navarro et al. 2000a,b) already revealed the occurrence of "anomalous" Stokes $V$ profiles during the flash events. The anomalous profiles could only be explained as the superposition of two regular profiles of opposite polarity and considerably Doppler-shifted with respect to each other. The obvious

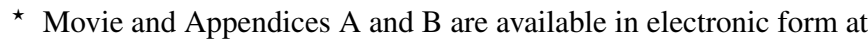
http: //wWw . aanda.org conclusion then is that, while UFs are a large-scale phenomenon, forming emission patches that are several arc-seconds across, they harbor fine-scale structure. Therefore, even inside a single pixel, one has a mixture in the horizontal direction where a certain filling factor is occupied by a "quiet" component (no reversal and zero or slightly downflowing velocity) while the rest is occupied by the shock-wave (line core emission reversal and strong upflows).

Additional indirect evidence supporting the two-component scenario described above was presented by Centeno et al. (2005) using observations of the He I $10830 \AA$ multiplet acquired with the TIP instrument (Collados et al. 1999). However, such fine structure inside the umbral flash patches was not directly seen until years later, with the analysis of Socas-Navarro et al. (2009) using high-resolution images of the $\mathrm{Ca}$ II $\mathrm{H}$ line core from the Hinode satellite (Kosugi et al. 2007; Tsuneta et al. 2008). With the increased spatial resolution offered today by some groundbased telescopes, it has been possible to confirm this finding by other authors (Bharti et al. 2013; Henriques 2013).

Umbral flashes seem to be connected to the phenomenon known as running penumbral waves, an oscillatory pattern originally observed in $\mathrm{H} \alpha$ images (but now also in He $\mathrm{I}$, as shown by Bloomfield et al. 2007, and Ca II in this study). The wave pattern is seen to move horizontally across the penumbra, running away from the umbra in the radial direction (Tsiropoula et al. 2000; Rouppe van der Voort et al. 2003). The relationship between UFs and penumbral waves is not only suggested by visual appearance. The first spectro-polarimetric observations of López Ariste et al. (2001) showed evidence that the shock bends 
radially outwards following the field lines as it propagates upwards. More recently, the inversions of infrared Stokes spectra carried out by Bloomfield et al. (2007) indicate that the same wave propagation that gives rise to the umbral flashes is at work in the penumbra and produces the running penumbral waves in a scenario where the magnetic field is more inclined.

The paper is arranged as follows: the observations and data processing are described in Sect. 2. A detail description of the umbral flashes in our observations and a simple analysis of the magnetic field strength, using the weak-field approximation are provided in Sect. 3. In Sect. 4 we describe the non-LTE inversion code NICOLE and our results are presented in Sects. 5 and 6. Finally, we summarize our conclusions in Sect. 7.

\section{Observations and data processing}

The data used for this work were acquired at the Swedish 1-m Solar Telescope (SST, Scharmer et al. 2003) using the CRisp Imaging SpectroPolarimeter (CRISP, Scharmer 2006; Scharmer et al. 2008). CRISP is a Fabry-Perot interferometer (FPI) consisting of two etalons mounted in tandem. With this kind of instrumentation one is able to record quasi-monochromatic images at a given wavelength of choice. A line profile may then be constructed by scanning the corresponding spectral range, obtaining images at each line position.

The observations were obtained on 2011 May 4 at 08:30 UT. The target was a sunspot (AR11204) at heliocentric $(x, y)=$ $\left(-336^{\prime \prime}, 332^{\prime \prime}\right)$, observing angle $30^{\circ}(\mu=0.87)$.

The dataset includes full-Stokes observations in Ca II $\lambda 8542$ and non-polarimetric images in $\mathrm{H}$ I $\lambda 6563(\mathrm{H} \alpha)$. The Ca II $\lambda 8542$ line was observed at 14 line positions with a sampling of $75 \mathrm{m \AA}$ between $\Delta \lambda= \pm 300 \mathrm{~m} \AA$ (an effective integration time of $900 \mathrm{~ms}$ ), and points at $\Delta \lambda= \pm 400, \pm 860,+3100 \mathrm{~m} \AA$ from line center (with an effective integration time of $210 \mathrm{~ms}$ ). A complete spectropolarimetric line-scan is acquired in approximately $16 \mathrm{~s}$. Images are acquired concatenating four polarization states. Each polarization state is a linear combination of the four Stokes parameters $(I, Q, U, V)$. The noise, relative to the continuum intensity, is $\sigma(Q, U, V)=(0.09,1.0,1.3) \times 10^{-3}$. The $\mathrm{H} \alpha$ line is observed at $\Delta \lambda=+0,+1300 \mathrm{~m} \AA$ from line center.

Figure 1 illustrates the observed line positions at 18542 , which are marked on a quiet-Sun spatial average from the surroundings of the sunspot. Ideally, one point in the continuum would be desirable but the presence of secondary transmission lobes in the CRISP transmission profile restricts the observations to the central part of the prefilter (see the bottom panel in Fig. 1).

The data processing was done following a similar approach as presented by Schnerr et al. (2011) but with some differences in the flat-fielding process, further discussed in Appendix A. High spatial resolution and precise alignment between the sequentially recorded images for different tunings was achieved with the multi-object-multi-frame-blind-deconvolution (MOMFBD, van Noort et al. 2005) technique for image restoration. The image scale is $0{ }^{\prime} 059$, which is optimal for observations at $\lambda 5500 \AA$. At longer wavelengths, the raw images are slightly oversampled, which is not ideal to maximize the signal-to-noise ratio at each pixel. However, this no longer true for the restored data since those are filtered at the resolution limit of the observations by the MOMFBD process.

In certain areas, where the scale of the seeing motions is smaller than the assumed size of the iso-planatic patch in the MOMFBD code, residual distortions can be present within the images of a same scan, or even the different polarization states
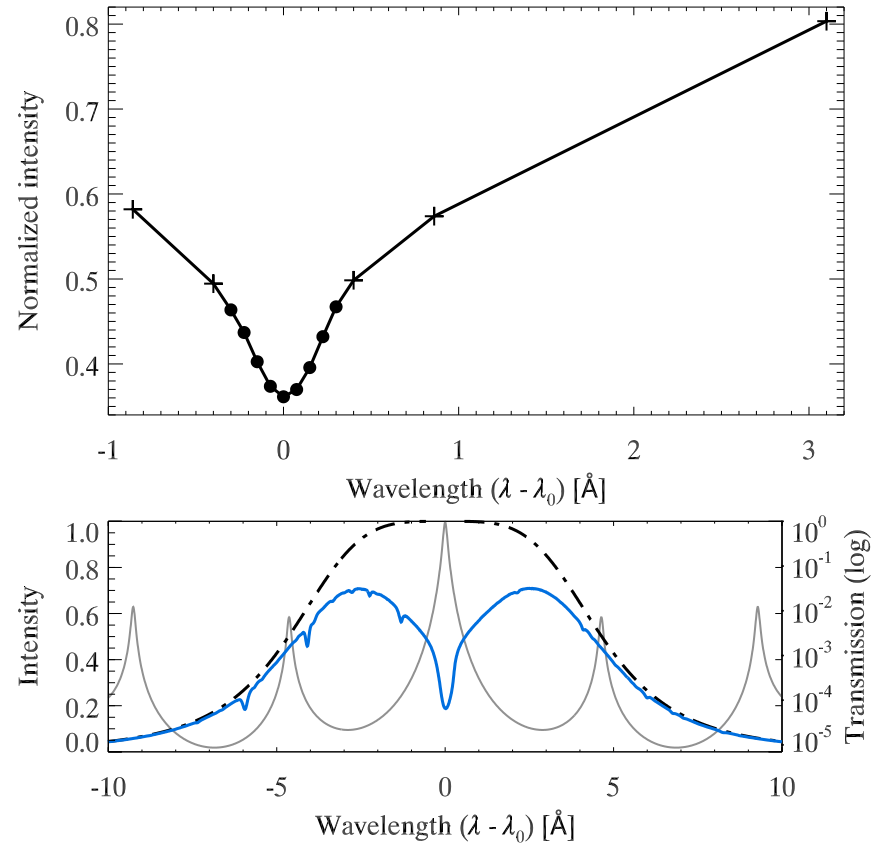

Fig. 1. Top panel: Ca II $\lambda 8542$ observations acquired at the line positions indicated on the line profile. Exposure time is $900 \mathrm{~ms}$ (circles) and $210 \mathrm{~ms}$ (crosses). Bottom panel: CRISP instrumental profile at $854.0 \mathrm{~nm}$ (solid gray) in logarithmic scale. A solar-atlas profile multiplied with the theoretical estimate of the prefilter (dashed-dotted line) is indicated in blue.

at a given wavelength. Reducing the patch size during the reconstruction can help to minimize this effect, but in some cases it does not remove it entirely. Henriques $(2012,2013)$ proposed to include extra wide-band images (one per wavelength and polarization state) that do not contribute to the wavefront-estimation in the MOMFBD process, but contain the same residual distortions as the narrow-band images. The distortions are measured by comparing those extra images with the anchor wide-band image and each narrow-band image is de-stretched accordingly.

The polarimetric calibration of the instrument was performed in 2D, resulting in a different Mueller matrix for each pixel (van Noort \& Rouppe van der Voort 2008). The telescopeinduced polarization was removed using the SST model developed by Selbing (2010) with the parameters fitted at $\lambda 8542$. The telescope model is further described in Appendix B.

The time series has been compensated for telescope rotation, alignment over time and residual rubber-sheet deformations (Shine et al. 1994) using the wide-band images as reference.

In order to normalize the data to a spatially-averaged quietSun continuum, we computed the intensity ratio between $\mu=$ 0.87 and $\mu=1.0$ at $\Delta \lambda=+3100 \mathrm{~m} \AA$ from line center, using synthetic profiles, computed in non-LTE, from a 3D hydrodynamical simulation (de la Cruz Rodríguez et al. 2011). This ratio was then applied to the intensity at the same wavelength of the McMath-Pierce Fourier Transform Spectrometer atlas (hereafter FTS atlas, Brault \& Neckel 1987), which has been previously convolved with the CRISP transmission profile. In this manner we impose that the spatially-averaged quiet-Sun profile at $\Delta \lambda=3100 \mathrm{m \AA}(\mu=0.87)$, must have an intensity of 0.792 relative to the continuum at $\mu=1$.

Finally, to compensate for the CRISP prefilter response, we compared the spatially-averaged quiet-Sun profile at solar-disk center, estimated from the flat-field data, with the solar atlas 
convolved with a theoretical CRISP profile. The parameters of a Lorentzian curve where iteratively fitted to reproduce the observed spectra. The theoretical prefilter curve is given by the following equation:

$$
P(\lambda)=\frac{1}{1+\left(2 \frac{\lambda-\lambda_{\mathrm{P}}}{\Delta \lambda_{\mathrm{P}}}\right)^{4}},
$$

where $\lambda_{\mathrm{P}}$ is the central wavelength of the prefilter and $\Delta \lambda_{\mathrm{P}}$ is the Full-width-half-maximum of the prefilter. These theoretical curves work particularly well with most CRISP prefilters (Schnerr et al. 2011; Scharmer et al. 2013).

Figure 2 summarizes our observations. In the photosphere (see the wide-band image in the top-right panel), photospheric granulation surrounds an irregular sunspot. It has developed penumbra in approximately $3 / 4$ of its circumference and a lightbridge divides the umbra in two halves (umbral cores). There is also a small pore on the top-left corner of the image.

The H $\alpha$ chromospheric image (top-left) is dominated by very dynamic fibrils which extend outwards, almost radially from the umbra-penumbra boundary. Some fibrils originate in the forementioned pore and are oriented towards the sunspot, suggesting some magnetic connection between the two.

The lowermost four panels correspond to the four Stokes parameters at $-150 \mathrm{~m} \AA$ from line center at $\lambda 8542$. The Stokes $I$ image is remarkably similar to that in the core of $\mathrm{H} \alpha$.

The Stokes $Q$ and $U$ signals, produced by magnetic fields perpendicular to the our line of sight, are mostly observed on the limb side of the sunspot, preferentially along elongated fibrils that extend in the superpenumbra (Bray \& Loughhead 1974). The Stokes $V$ image (associated with the longitudinal magnetic field component) shows very strong dynamics, with frequent sign changes due to emission reversals. The time series shows that UFs and running penumbral waves are present in the sunspot on both sides of the light-bridge.

We have made extensive use of CRISPEx (Vissers \& Rouppe van der Voort 2012), a tool for the exploration of multidimensional CRISP datasets.

\section{Umbral flashes at high-spatial resolution}

The $\lambda 8542$ data presented here are, to our best knowledge, the highest-resolution spectroscopic observations of UFs. Figure 3 shows the detailed evolution of a subfield (marked in Fig. 2 with the white square in the lower right corner) for eight time steps of our series. In Stokes $I$ (first and second row), signatures of the UF are visible from $\Delta t=32 \mathrm{~s}$ to $\Delta t=80 \mathrm{~s}$, and the sunspot seems to be in a quiescent state everywhere else. During the UF, our images reveal dynamic fine structure within the umbra that resemble penumbral micro-jets (Katsukawa et al. 2007), similarly reported by Socas-Navarro et al. (2009).

In Stokes $V$, the imprint of the UF is dramatic. During quiescence, the Stokes $V$ images show negative polarity everywhere in the umbra. As the flash starts at $\Delta t=32$, the presence of polarity changes is tightly correlated with brightenings in Stokes $I$.

Our observations also show the imprint of micro-jets above the penumbra in Stokes $V$. They are seen very conspicuously when the time series is played as a movie.

The Stokes $Q$ and $U$ signals are too weak inside the umbra. However, the fibrils that form the superpenumbra on the limb side of the spot, show strong signal in Stokes $Q$ and $U$ within $\Delta \lambda= \pm 300 \mathrm{~m} \AA$. Running penumbral waves are visible in the four Stokes parameters. In Sect. 6 we analyze them in more detail.

\subsection{The weak-field approximation for Zeeman-induced polarization}

The weak-field approximation provides a relatively simple way of computing the magnetic field vector from full-Stokes observations (see Landi Degl'Innocenti \& Landolfi 2004, and references therein). It works particularly well in those cases where the Doppler width of the observed line $\left(\Delta \lambda_{D}\right)$ is larger than the Zeeman splitting produced by the magnetic field $\left(\Delta \lambda_{B}\right)$. Under these conditions, the observed Stokes $Q, U$ and $V$ profiles can be reproduced as a function of the magnetic field strength and the first derivative of the Stokes I profile.

The Ca II $\lambda 8542$ line has a relatively low Landé factor $(\bar{g}=$ 1.10) and it is broader than most photospheric lines. Therefore, the weak-field approximation can be safely used even when the magnetic field is relatively strong. The inequality $\Delta \lambda_{\mathrm{D}}>\Delta \lambda_{B}$ provides a convenient means to calculate the regime of applicability for the approximation (Asensio Ramos 2011):

$B<\frac{4 \pi m_{\mathrm{e}} c}{\bar{g} \lambda_{0} e_{0}} \sqrt{\frac{2 k T}{M}+v_{\mathrm{mic}}^{2}}$,

where $m_{\mathrm{e}}$ and $e_{0}$ are the electron charge and mass, $\lambda_{0}$ is the central wavelength of the line, $c$ is the speed of light, $k$ is the Boltzmann constant, $M$ is the mass of the atomic element, $T$ is the temperature and $v_{\text {mic }}$ the microturbulence.

Assuming a chromospheric scenario with $T=4500 \mathrm{~K}$, $v_{\text {mic }}=3 \mathrm{~km} \mathrm{~s}^{-1}$ and calcium atoms, the upper limit is approximately $B \leq 2500 \mathrm{G}$. Even though this value is lower than usual photospheric values in sunspots $(B \approx 3000 \mathrm{G})$, the magnetic field strength is usually weaker in the chromosphere.

One limitation of the weak-field approximation is that it also assumes a constant magnetic field. While most photospheric lines are sensitive to a relatively thin layer of the atmosphere, where the magnetic field is nearly constant, the $\lambda 8542$ line is sensitive to a range of approximately $1500 \mathrm{~km}$ (e.g. Cauzzi et al. 2008) and it is less justified to assume a constant magnetic field over that height range. Another possible approach would be to use the bisector method (Rayrole 1967; Landi Degl'Innocenti \& Landolfi 2004), which allows one to retrieve some depth information but it is more sensitive to noise.

In this analysis we use the weak-field approximation to infer an approximation to the line-of-sight (l.o.s.) magnetic field strength at chromospheric heights. To keep a simple approach, we use only $\Delta \lambda= \pm 225 \mathrm{~m} \AA$ from line center, where the images are clearly dominated by chromospheric features ("fibrils", umbral flashes), and photospheric granulation is not visible.

Figure 4 summarizes our results for one pixel in the sunspot as a function of time. The top row illustrates $t-\lambda$ diagrams for Stokes $I$ and $V$. The rightmost panel shows the Stokes $V$ profiles that result from our fits using the weak-field approximation.

The figure shows nicely the Stokes $V$ polarity reversals during the UF, which coincide with emission in the Stokes $I$ profile. In fact, the shapes of the observed Stokes $V$ profiles are tightly reproduced by the first derivative of Stokes $I$ and the polarity reversals are consistent with the changes in the slope of Stokes $I$ that are induced by the UF.

The top-middle panel in Fig. 4 illustrates the inferred longitudinal magnetic field component during the time series. Despite some fluctuations with time, the magnetic field strength remains rather constant showing that it is possible to reproduce UF profiles with a constant magnetic field. We have indicated all the UF phases using short vertical lines at the bottom of the panel.

For clarity, we have selected one quiescent profile and one UF profile, marked in the top row with black and blue horizontal 

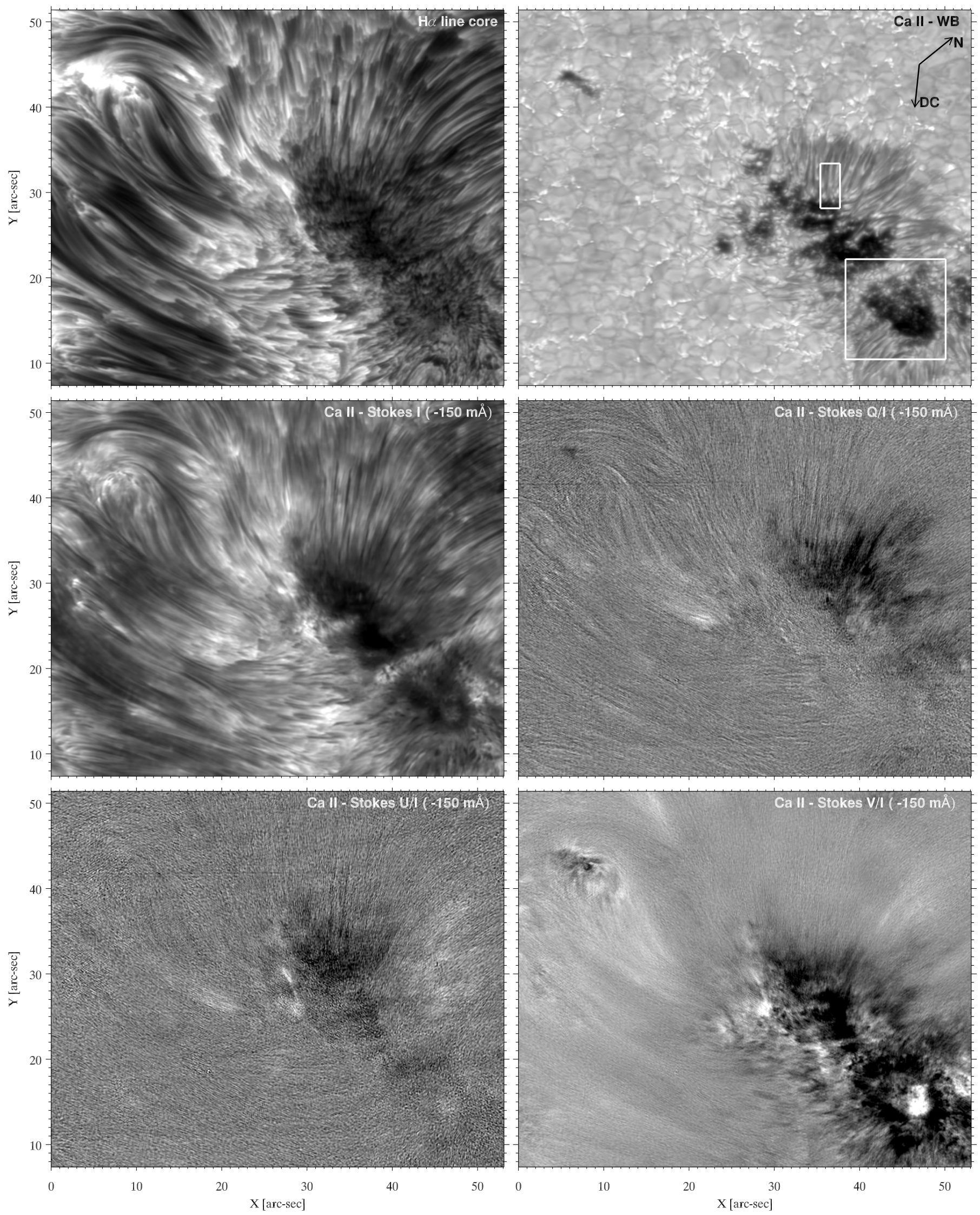

Fig. 2. CRISP filtergrams at the core of the $\mathrm{H} \alpha$ line (top left). All other images are in Ca II $\lambda 8542$. A wide-band image showing the photosphere is displayed in the top right panel. The lowermost four panels show the chromosphere at $\Delta \lambda=-150 \mathrm{~mA}$ from line core in Stokes $I$ (middle left), $Q / I$ (middle right), U/I (bottom left) and V/I (bottom right). The arrows on the wide-band image indicate the direction to the solar North and to disk center, respectively. The largest white box indicated in the wide-band image highlights the subfield that has been used for the non-LTE inversions, whereas the smaller square indicates a subfield used to study running penumbral waves. 
J. de la Cruz Rodríguez et al.: Physical properties of a sunspot chromosphere with umbral flashes
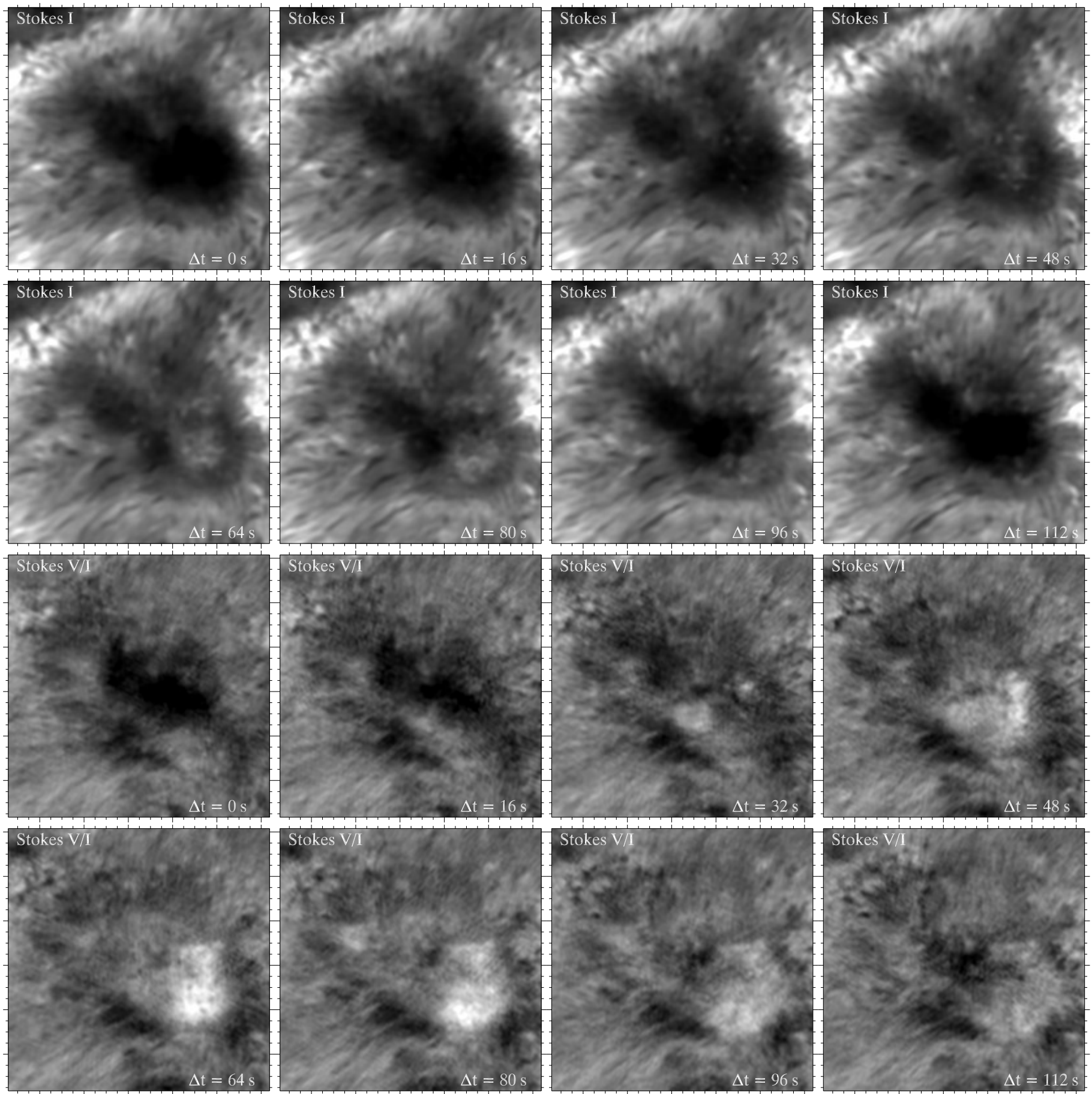

Fig. 3. Time-series in Ca II $\lambda 8542$ showing a subfield of the FOV in Stokes $I$ (the two uppermost rows) and Stokes $V / I$ (two lowermost rows) at $\Delta \lambda=-150 \mathrm{m \AA} . \Delta t=0$ occurs at 09:01:48, and the UF is clearly visible in the central part of the series $(\Delta t=48-80 \mathrm{~s})$. The minor tickmark separation is 0.5 . An online movie is available.

lines, respectively. Both profiles are plotted in the lowermost two panels. The corresponding time steps are marked in the uppermiddle panel, showing that the field strength is very similar during the quiescent and flash phases (at least within the limitations of the weak-field approximation which provides us with a rough average of the magnetic field).

These results confirm that the strong variability of the $\lambda 8542$ line is likely to be produced by changes in the temperature and velocity of the plasma and, to a much lesser extent, by changes in the magnetic field. Note that the field is probably perturbed by the shock waves during the UF, but it is not a main contributor to that variability observed in Stokes $I$ or $V$.

\section{The inversion code NICOLE}

To derive atmospheric parameters from our spectropolarimetric observations, we use the non-LTE inversion code NICOLE (Socas-Navarro et al. 2000c, and in prep.). NICOLE solves the non-LTE problem using preconditioning as explained by Socas-Navarro \& Trujillo Bueno (1997), assuming that each 

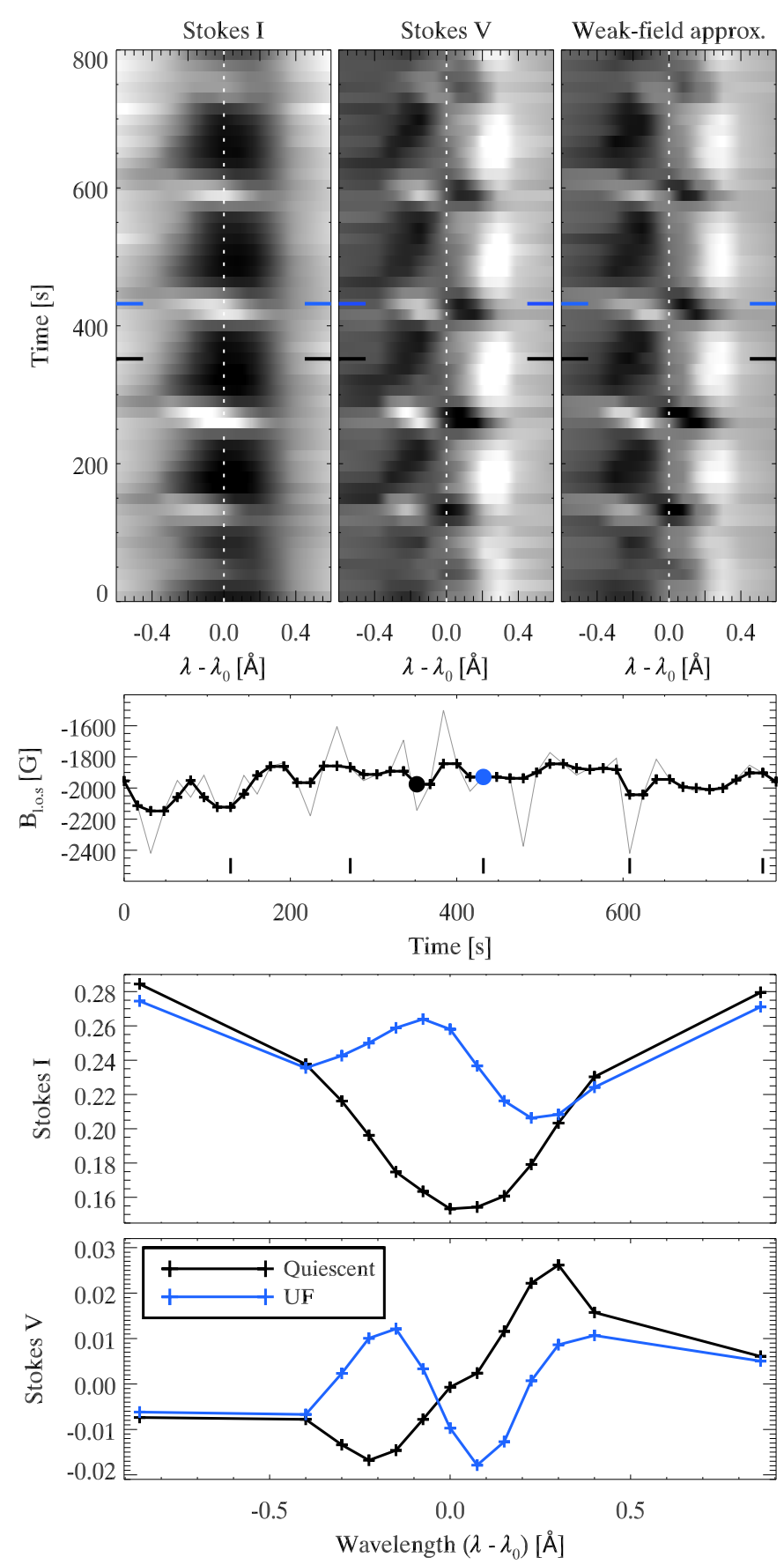

Fig. 4. Time-series at a single pixel within the sunspot is shown in the top three-panels, in Stokes I (top left) and $V$ (top middle). The top-right panel corresponds to a synthetic Stokes $V$ profile computed using the weak-field approximation to fit $B_{\text {l.o.s }}$. In both cases, the Stokes $V$ profiles are scaled identically between $\pm 2.2 \%$ relative to the average quiet-Sun continuum intensity. The resulting longitudinal magnetic field strenght is plotted in the second row (solid-grey line). We have smoothed that result using a median filter of 3 time-steps, which is illustrated with a black-crossed line. For clarity, the lowermost panels illustrate the Stokes $I$ and $V$ profiles at a quiescent state (black) and during the UF (blue).

pixel harbors a plane-parallel atmosphere. All Zeeman sublevels originating from a given atomic level are assumed to be equally populated, discarding any quantum interference between them, as proposed by Trujillo Bueno \& Landi Degl'Innocenti (1996). We use a Ca II model atom consisting of five bound level plus a continuum (see Leenaarts et al. 2009) and the collisional broadening is computed according to Barklem \& O'Mara (1998).

The velocity-free approximation is used to compute the atom population-densities. By neglecting the velocity-field, only half of the profile needs to be computed and fewer quadrature angles are used to compute the mean intensity at each depth-point. This approximation is justified by the very broad shape of the $\mathrm{Ca}$ II lines and the fact that the atomic level populations are dominated by the radiation field at the line core. Once the atomic populations are converged, the emerging full-Stokes vector is computed including the velocity field using a quadratic DELOBezier formal solution of the polarized radiative transfer equation (de la Cruz Rodríguez \& Piskunov 2013).

Scattering polarization is not included in our calculations but this is not a problem because the magnetic field is sufficiently strong to produce Zeeman-dominated Stokes profiles. In the $\lambda 8542$ line, it is safe to assume Zeeman induced signal when the magnetic field is stronger than approximately $100 \mathrm{G}$ (further details can be found in Manso Sainz \& Trujillo Bueno 2010; Carlin et al. 2013).

NICOLE has been previously used in LTE studies (Socas-Navarro 2011; Scharmer et al. 2013) and validated in non-LTE using a chromospheric 3D MHD numerical simulation (de la Cruz Rodríguez et al. 2012). In that study, the synthetic profiles are degraded with a realistic CRISP transmission profile. In order to properly convolve with the instrumental profile, the synthetic spectra are calculated first in a fine grid of regularly spaced wavelength points. The inversion code works internally with such finely sampled profiles except when it comes to comparing with observations, at which point only the observed line positions are used to drive the inversion.

The inversion process is initialized with a smoothed version of the HSRA atmosphere (Gingerich et al. 1971). Node points are located equidistantly along the depth-scale, where corrections to the model are applied. The correction in between nodes is determined as a cubic Bezier-spline interpolation (see Auer 2003) of the node values.

To improve convergence properties, the inversion is run in two cycles as proposed by Ruiz Cobo \& del Toro Iniesta (1992). In the first cycle, fewer degrees of freedom (nodes) are prescribed. Once the solution cannot be further improved, a new cycle is started with an (slightly) increased number of nodes. The degrees of freedom used for each cycle, summarized in Table 1, have been obtained after some trial-and-error experiments with a few sample profiles. Note that we have used fewer nodes than in de la Cruz Rodríguez et al. (2012) because our observations are slightly undersampled around line core, and very few points are present in the photospheric wings of the line. Therefore, some depth information is lost with respect to what could be obtained with more observables.

The equation of state defines a unique relationship among temperature, electron pressure and gas pressure. If two of them are known, then the third one can be obtained from it. During the inversion, however, only the temperature is set for any guess model. Therefore, hydrostatic equilibrium is used to compute the gas pressure.

\section{Atmospheric properties during umbral flashes}

To characterize the atmospheric state during UFs, we have inverted two time steps from the series, corresponding to $\Delta t=16 \mathrm{~s}$ and $\Delta t=64 \mathrm{~s}$ in Fig. 3. To accelerate the computations, only every second pixel in both spatial directions was inverted. We 
Table 1. Summary of node points used during each cycle of the inversion.

\begin{tabular}{lcc}
\hline \hline Physical parameter & Nodes in cycle 1 & Nodes in cycle 2 \\
\hline Temperature & 4 & 5 \\
l.o.s velocity & 2 & 3 \\
Microturbulence & 0 & 0 \\
$B_{\text {l.o.s }} \equiv B_{z}$ & 0 & $1 \& 2$ \\
$B_{x}$ & 0 & 1 \\
$B_{y}$ & 0 & 1 \\
\hline
\end{tabular}

Notes. $B_{\text {l.o.s }}$ is oriented along the $z$-axis.

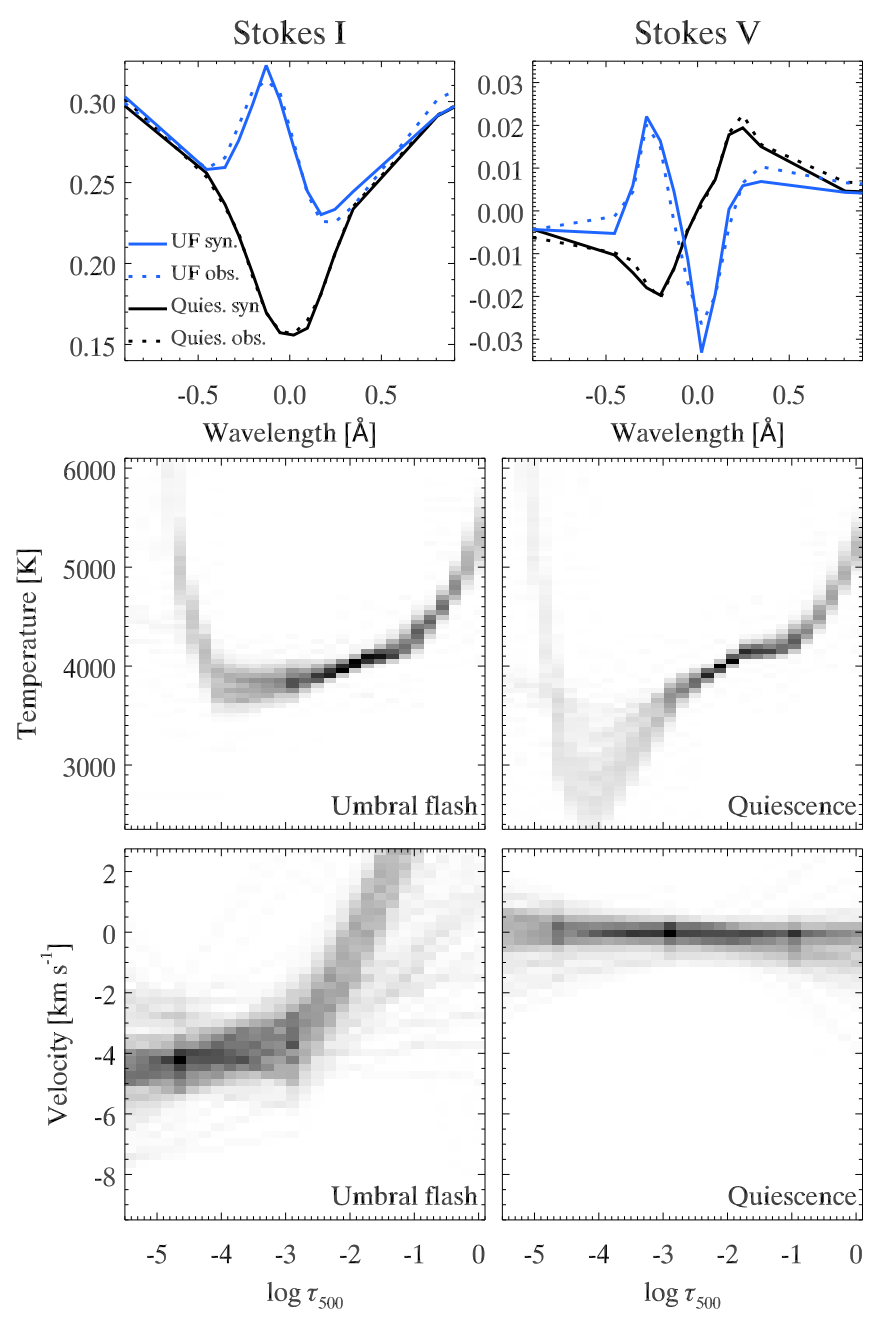

Fig. 5. Top row: observed (dashed line) and inverted (solid line) profiles from one pixel within the sunspot during the UF phase (blue) and quiescence (black). Middle row: density map illustrating the inferred temperature stratification during UF (left) and quiescence (right) for a patch inside the umbra. A total of 280 models is shown. Bottom row: same as middle row, for the l.o.s velocity stratification. Negative velocities correspond to upflows.

are not interested in the very small-scale (close to the telescope diffraction limit) details of the umbra. Therefore, this loss of resolution (now 0.'24) does not influence our conclusions significantly whereas computation speed is decreased by a factor of four.

Fits to sample profiles are shown in Fig. 5. Observed (dashed line) and synthetic (solid line) profiles are plotted for a quiescent phase (black line) and during a UF (blue line) in Stokes $I$ (left) and $V$ (right). Stokes $Q$ and $U$ profiles are too noisy to obtain meaningful fits in the center of the spot. The inversion code is able to reproduce to a large extent the spectral features present in the observations, including the emission reversal seen at line center during the UF. The middle and bottom rows show the inferred quantities as a function of depth, within a $1^{\prime \prime} .75 \times 2$.' 35 patch around the point marked with the cross in Fig. 6, which are discussed below along with 2D maps of the inferred quantities.

In interpreting inversion results, it is important to understand that we cannot "see" a discrete height. If we take an atmosphere and apply a perturbation (e.g., increase the temperature) in a slab that is much narrower than the photon mean free path, then the perturbed slab would be completely transparent and the radiation would travel through it unchanged. Thus, it is impossible to determine observationally the properties of a very thin layer. The inversion is only to the average properties of the atmosphere on length scales that are long enough to leave an imprint on the emerging radiation. This is not a limitation of the inversion technique or the instrumentation. It is an intrinsic limitation imposed by the integral form of the radiative transfer equation. How severe this limitation is in practice, depends on a number of parameters, the most important being the number of spectral lines observed. Obviously, having several lines probing the same atmospheric region increases the depth resolution of the inversions.

Because of the limitation in height resolution discussed above, it is not always sensible to interpret inversion results at a specific height. Instead, one needs to average in the vertical direction over a slab that is thick enough to have a measurable effect on the observed profiles. Therefore, the 2D maps presented in this section have been averaged over a depth range of 0.5 dex (decimal exponent), centered at the $\log \tau_{500}$ value indicated in each of the panels.

The temperature stratification within the umbra changes dramatically from the quiescence to the UF phase. The results from our inversions are illustrated using 2D maps at several optical depths in Fig. 6. Note that a constant $\tau$ slice usually maps a corrugated height surface.

In the middle photosphere, at $\log \tau_{500}=-0.5$, there is not a significant difference between quiescence and UF (lowermost panels of Fig. 6). We can only see those intensity changes produced by solar evolution. Some umbral dots are visible within the umbra, and part of the light bridge is included in the upperleft corner of the field of view (FOV).

In the upper photosphere (middle panels), the light bridge and surrounding penumbra become smoother and most of the fine structure of umbral dots has disappeared.

Signatures of the UF start to appear within the sunspot at approximately $\log \tau_{500}=-3.0$, where the temperature starts to increase relative to the quiescent phase. This temperature excess is very conspicuous at $\log \tau_{500} \approx-4.5$. The middle row in Fig. 5 shows that the photospheric temperature is essentially identical during both phases, starting to diverge approximately at $\log \tau_{500}=-3$. The distributions of models in this small subfield shows that the maximum temperature difference between both phases, about $1500 \mathrm{~K}$, occurs at $\log \tau_{500}=-4$.

The $2 \mathrm{D}$ temperature maps in the chromosphere (top row in Fig. 6), show a hot canopy above the umbra during the UF that is not present during quiescence. This canopy coincides with those areas showing enhanced line-core emission.

When shocks are present, discontinuities are present in the vertical direction of some physical parameters. Those are blurred out by the radiative transfer and, as discussed above, observations do not allow to distinguish a sharp change from a smoother 


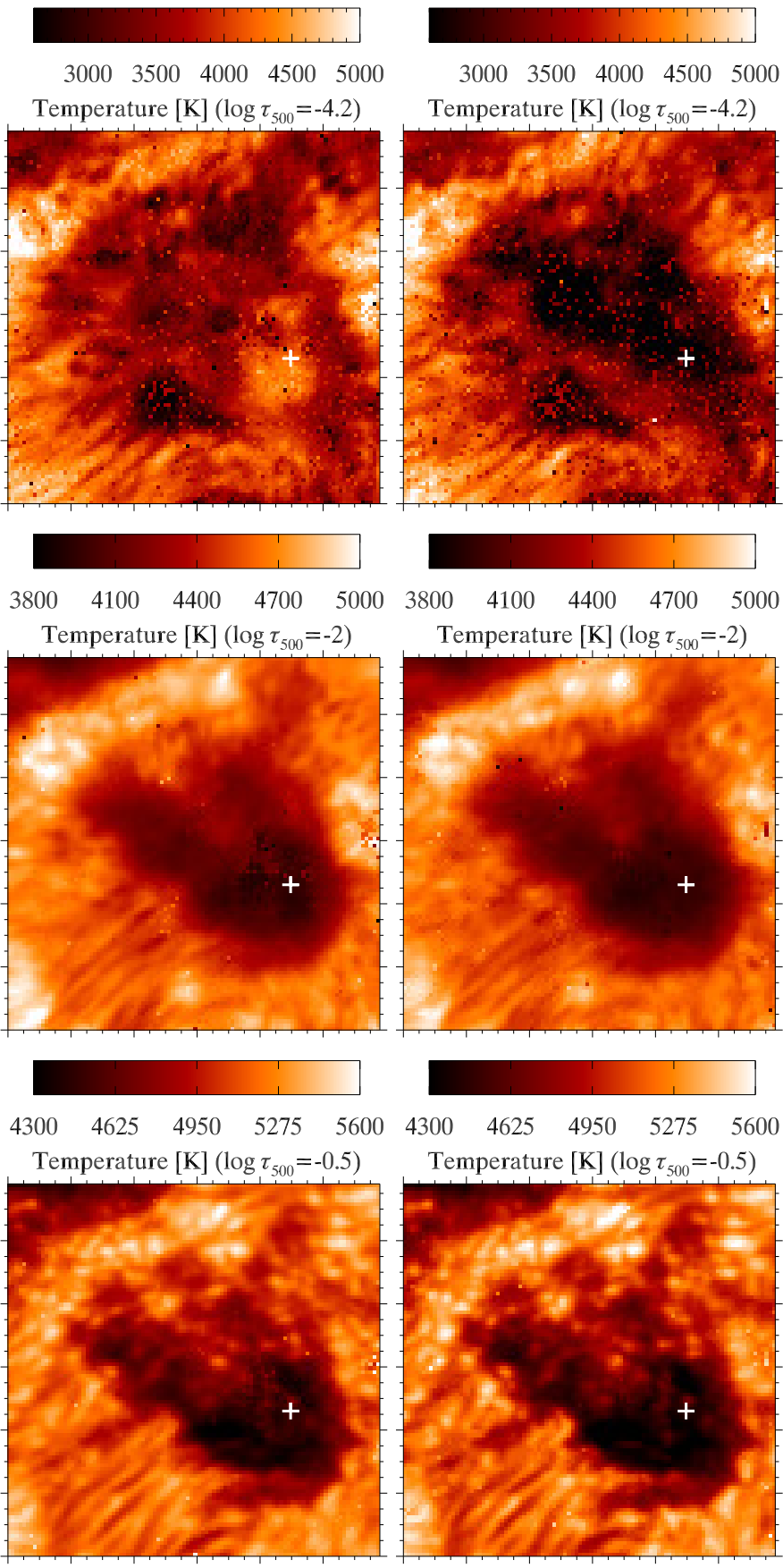

Fig. 6. Inferred temperature from a non-LTE inversion. Temperature slices from the inversion are shown in two columns at quiescent (right) and flash (left) phases at several optical-depth $\log \tau_{500}=-4.2,-2,-0.5$ respectively from top to bottom. Note that we have performed a depth average of $0.5 \mathrm{dex}$, centered at those heights. The minor tick-mark separation is 0.5 . The white cross marks the center of the patch of which the temperature and velocity stratifications are shown in Fig. 5

transition. (de la Cruz Rodríguez et al. 2012) demonstrated how in similar situations, inverting only the $\lambda 8542$ line, the inversion retrieves a somewhat smoothed out version of the shock.

Figure 7 shows the 1.o.s velocity component at $\log \tau_{500}=$ -4.2 and $\log \tau_{500}=-2$ from top to bottom respectively. In the chromosphere (top row), the inversion code reconstructs upflowing velocities up to $5 \mathrm{~km} \mathrm{~s}^{-1}$ in the flash (left panel). During the quiescence, the umbra shows an homogeneous downflow patch $\left(\sim 1 \mathrm{~km} \mathrm{~s}^{-1}\right)$, similarly to the scenario reported by Socas-Navarro et al. (2001).
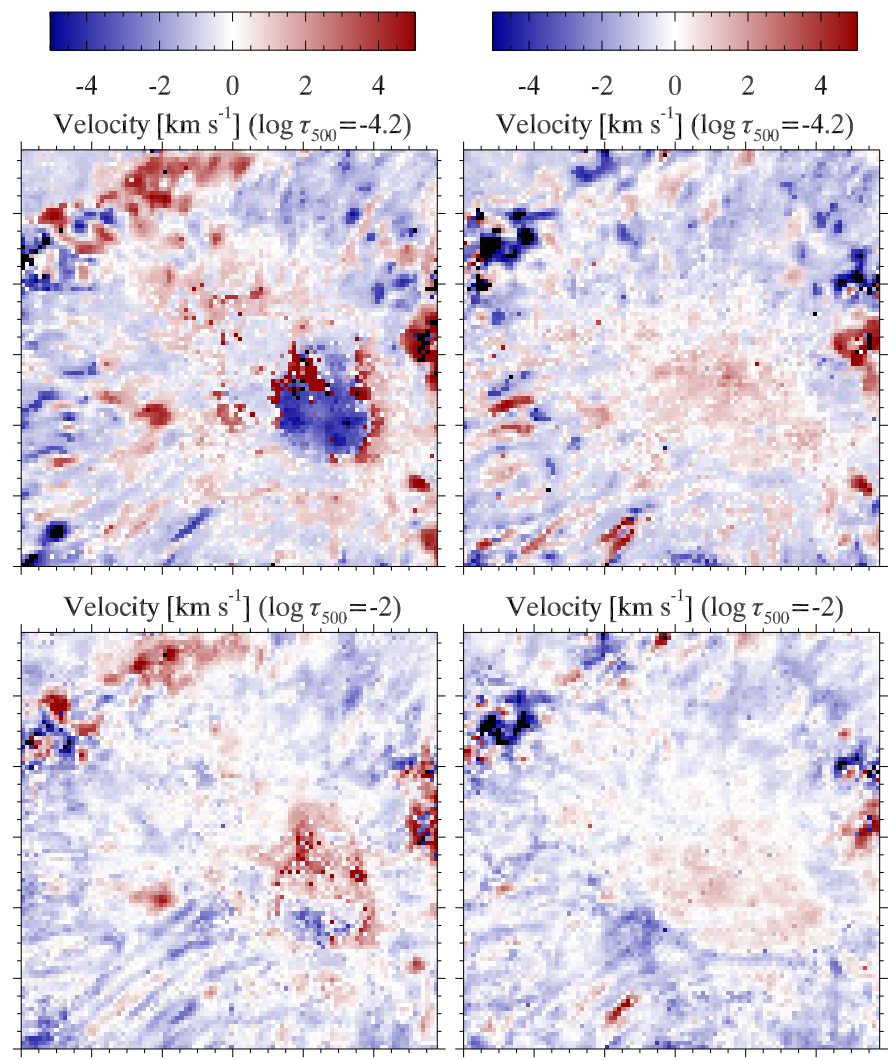

Fig. 7. 1.o.s. velocity component from a non-LTE inversion. Slices at constant optical depth are shown in two columns at quiescent (right) and flash (left) phases at $\log \tau_{500}=-4.2,-2$ respectively from top to bottom. Negative velocities correspond to upflows. The minor tick-mark separation is 0.5 .

Another interesting result is found on the light bridge. The time series shows that micro-jets have apparent up and down motions in the intensity images. Our maps show that those motions are in counter-phase with the UF within the umbra, suggesting that the flash has an effect on the motions of the light bridge, as well.

One limitation of our observing setup is that we have only one pseudo-continuum point in the far wing of the line. This point allows us to probe the lower photospheric temperature but, lacking a symmetric point on the other side of the line, the velocities at that height are poorly determined. This explains why the temperature map is smooth in the photosphere whereas the corresponding velocity map is much noisier.

The longitudinal component of the magnetic-field reconstructed by the inversion is noisy. We have compared the results from inversions with 1 node and 2 nodes respectively in $B_{\text {long }}$. The resulting maps are almost identical when a depth average between $\log \tau_{500}=-4.5$ and $\log \tau_{500}=-3.5$ is considered, although we notice that the inversion noise increases when the degrees of freedom is higher.

Further testing with the inversion weights (different for each of the Stokes parameters) showed that the reduced wavelength coverage in our observations allows the code to over-fit the noisy Stokes $V$ profiles, which are usually perfectly reproduced by the fitted spectra ${ }^{1}$.

1 We plan to acquire new observations where the line profiles are critically sampled and investigate this issue by systematically dropping more line positions to calculate the inversion. 

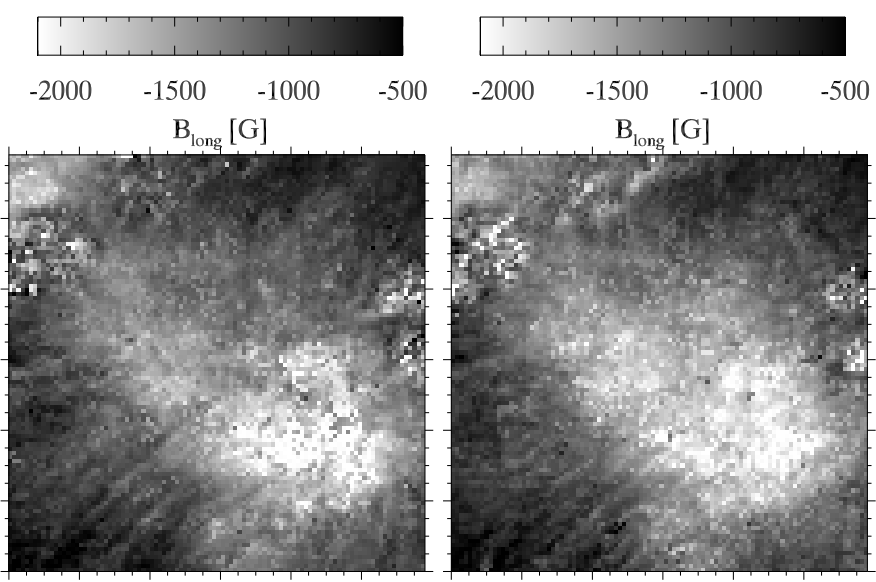

Fig. 8. Longitudinal magnetic field component from a non-LTE inversion with one node in $B_{\text {long }}$, during the UF phase (left) and quiescence (right). The minor tick-mark separation is $00^{\prime} 5$.

Increasing the degrees of freedom makes this situation worse, therefore we have used the inversion including only 1 node, illustrated in Figs. 8 and 9. The former illustrates the resulting $B_{\text {long }}$ across the FOV, assuming a constant magnetic field as a function of depth. As expected, the magnetic field strength is stronger in the center of the umbra, and becomes weaker outwards, rather typical configuration for this kind of sunspots. Some fibril-like features are visible in the lower-left corner of the FOV, where the superpenumbra starts.

Even though we do not find significant changes in the configuration of the magnetic-field $\left(B_{\text {long }}\right)$ during the flash, small scale variations of approximately $200-300 \mathrm{G}$ are present within the umbra. Those variations would be compatible with a noise of $10^{-2}$ in Stokes $V$ (a rough estimate computed with the weakfield approximation, the FTS atlas profile and a magnetic field of $2000 \mathrm{G}$ ), however, our observations reach $10^{-3}$ in the range $\Delta \lambda= \pm 300 \mathrm{~m} \AA$, suggesting that the source of these variations may not be the noise.

Additionally, small scale features are present over the lightbridge (top-left corner), indicating a less smooth and more confined magnetic field configuration, suggesting a connection with the chromospheric jets that here are also present over the lightbridge.

Figure 9 illustrates a probability diagram comparing $B_{\text {long }}(\mathrm{UF})$ vs. $B_{\text {long }}$ (quiescence) over the inverted FOV. This figure is compatible with an scenario where the magnetic field does not change much during the UF, as we could somewhat conclude from our simple analysis using the weak-field approximation.

The lack of signal in Stokes $Q$ and $U$ inside the umbra does not allow for a relevant interpretation of the horizontal components of the magnetic field, however, we include 1 node in $B_{x}$ and in $B_{y}$ respectively so the code can compute a Zeeman splitting compatible with the observations where the magnetic field strength is close to $2500 \mathrm{G}$ (see Sect. 3).

\section{Running penumbral waves}

de la Cruz Rodríguez et al. (2012) justify and demonstrate the suitability of LTE inversions to derive the l.o.s. velocity and the magnetic field vector from chromospheric observations, disregarding the retrieved "temperature" merely as a fitting parameter parameter with no actual physical meaning. The idea is to allow the inversion code to adjust the temperature stratification

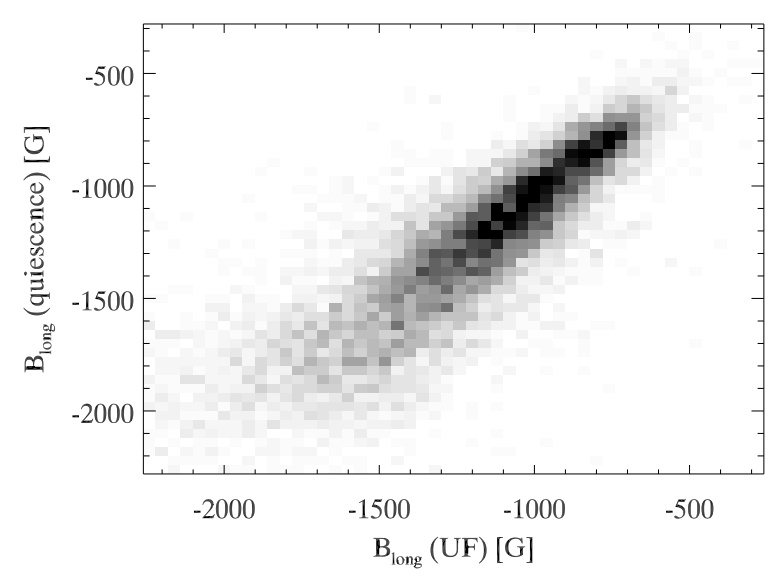

Fig. 9. A 2D probability map illustrating the relation between the longitudinal magnetic field component during the UF and quiescence, over the inverted FOV.

in such a way that the resulting LTE source function is the same that would be obtained in non-LTE with the correct temperature. Normally, in Ca II inversions this results in an unrealistically cold atmosphere lacking a transition region. The advantage of this approach is that one can then analyze longer time-series or larger FOVs as the computing time per inversion is significantly reduced.

Note that Beck et al. (2013) also perform LTE inversions to retrieve atmospheric parameters in the upper photosphere using the extended wings of the Ca II $\lambda 3968$ ( $\mathrm{H}$ line), but those authors restricted themselves to photospheric quantities.

We have used LTE inversions to study running penumbral waves in our data. With a similar setup to the one described in Sect. 4, we inverted a small subfield over 193 snapshots. The FOV has been selected to include a region with strong Stokes $Q$ and $U$ signal (thus improving our sensitivity to the transverse field component). The FOV is indicated in Fig. 2 (see the small white box on the WB image). This 2 . $5 \times 5^{\prime \prime}$ patch has been conveniently selected such that the running penumbral waves propagate parallel to the longest dimension.

The resulting magnetic field vector has been converted into the solar reference frame to compute the local inclination of the field (similarly done in Scharmer et al. 2013). We solve the $180 \mathrm{deg}$ ambiguity by selecting, at each pixel, the direction that makes the field point radially outwards in the local frame. In Figs. 10 and 11 we average the plotted quantities in the direction perpendicular to the wave propagation (the small dimension of the box) with the aim of increasing the signal-to-noise ratio. The 1.o.s. velocity and longitudinal magnetic field are depthdependent. For those quantities we plot depth averages from $\log \tau_{500}=-3.5$ to $\log \tau_{500}=-4.5$.

The top panels of Fig. 10 show the 1.o.s velocity (left), magnetic field strength (middle) and the magnetic field inclination (right) as a function of time. Waves are visible in these diagrams as a modulation pattern of alternating bright and dark features. In the penumbra (left part of the panels), these features are slanted which is indicative of wave propagation. These are the running penumbral waves which are so prominently visible in the Stokes $I$ and $V$ movies. In the umbra $(x>5)$ the modulation pattern is more horizontal and is related to UFs. The slanted features are clearly visible in the l.o.s. velocity and, with somewhat lower contrast, in the longitudinal field as well. The slope of the features indicate a propagation speed of about $13 \mathrm{~km} \mathrm{~s}^{-1}$, which is higher but consistent with the measurements of 


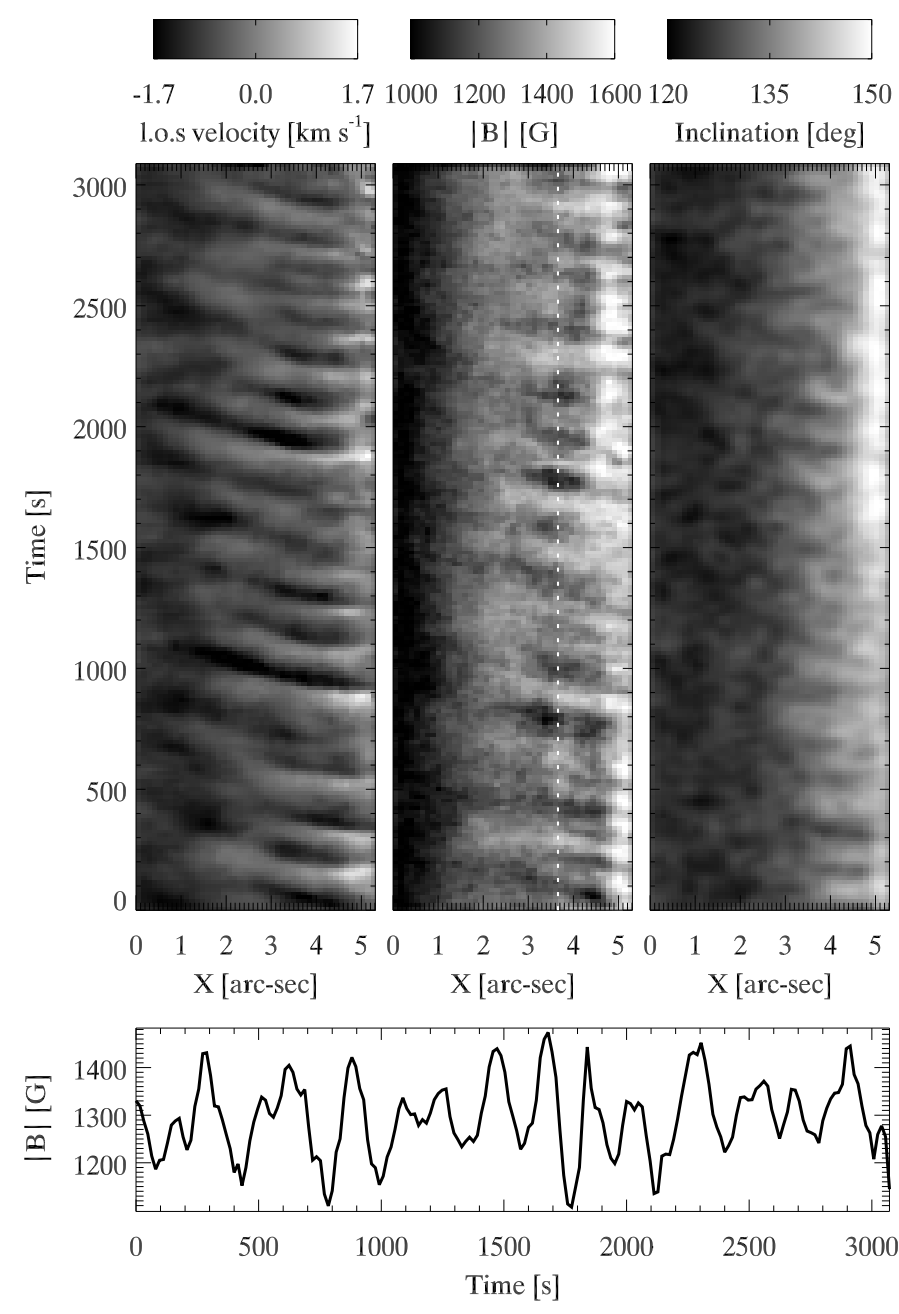

Fig. 10. $x$ - $t$ diagrams for the 1.o.s velocity (left), the magnetic field strength (middle) and the magnetic field inclination (right) are shown in the top row. The umbra is on the right side of the panels, the outer penumbra on the left side $(x=0)$. The detailed magnetic field strength modulation for one spatial location (at $x=3.65$, indicated with the white dotted line in the middle panel) is plotted in the bottom panel. The magnetic field inclination has been smoothed with a 3 pixels fullwidth-half-maximum Gaussian kernel.

Rouppe van der Voort et al. (2003) who analyzed running penumbral waves in Ca II $H$ and $K$ images.

According to the results shown in the middle panel, the magnetic field strength is perturbed by the running penumbral wave, showing peak-to-peak variations up to $200 \mathrm{G}$. For clarity, the time series along the white dotted line is shown at the bottom panel.

The rightmost panel in Fig. 10 illustrates the evolution of the magnetic field inclination as a function of time. The magnetic field is almost vertical close to the umbra and it rapidly becomes more horizontal outwards. Some modulation may be discerned as a function of time, but it is very weak and the fore-mentioned diagonal slope in the pattern is not clear. We then conclude that the field inclination is not perturbed significantly within our detection threshold (a few degrees) as the wave propagates. This result suggests that the shock front has an effect on the field strength but not on its orientation.

The FFT power spectra along each of the columns of the 1.o.s velocities and magnetic field strength (see Fig. 10) are presented in Fig. 11. We find that in both cases, higher frequencies

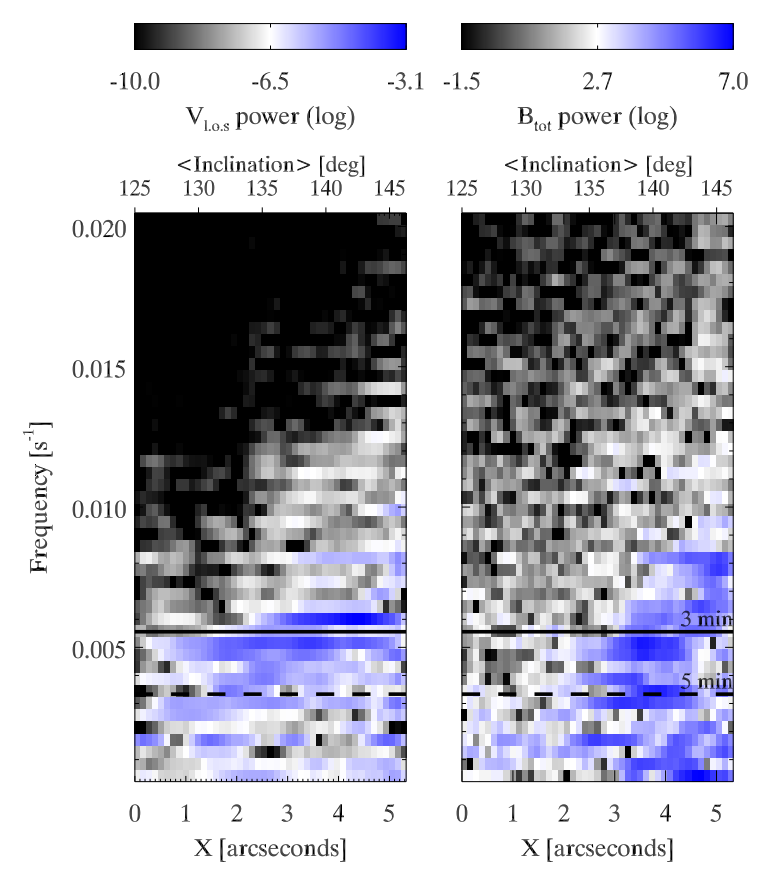

Fig. 11. FFT power spectra along each column for the 1.o.s velocities and magnetic field strength presented in Fig. 10. The axis labeling at the top indicates the average magnetic field inclination obtained through averaging over the right panel of Fig. 10. More inclined field is found in the outer penumbra $(x=0)$, more vertical field in the umbra (right side of the panels). The solid horizontal line indicates the 3 min mode, whereas the horizontal dashed line indicates the 5 min mode.

are more dominant towards the umbra (right side of the panels) where the magnetic field is more vertical (as indicated by the average magnetic field inclination derived from averaging over columns of the right panel of Fig. 10 and marked on the top axis). Outwards into the penumbra (lower values of $x$ ), most power is concentrated in lower frequency modes, producing a slope in the power spectra diagram.

\section{Conclusions}

In this paper we present a novel analysis of state-of-the-art spectropolarimetric observations of a sunspot chromosphere. In particular, we have focused on the study of the physical parameters of the chromosphere during UFs.

A simple analysis of the Stokes $V$ reversals that are observed during UFs show that, even with a nearly constant magnetic field, the profiles can be successfully reproduced. Despite the limitations of the weak-field approximation, it is possible to connect the variability of Stokes $V$ with the changes of the intensity profile (Stokes $I$ ).

The resulting model from our non-LTE inversions suggest a temperature excess up to $1000 \mathrm{~K}$ in the shocked chromosphere, relative to the quiescence phase. In the photosphere, the temperature stratification seems unaffected by the UF phenomenon.

To reproduce the observed profiles, a steep velocity gradient is reconstructed at $\log \tau_{500} \approx-3$. Above, the velocity remains almost constant around $v_{\text {l.o.s }}=4.5 \mathrm{~km} \mathrm{~s}^{-1}$. These velocities are similar to those from the simulation by Bard \& Carlsson (2010) at approximately $1000 \mathrm{~km}$ height.

In the photosphere, the line is very broad and our observations have limited coverage of photospheric wavelengths. Therefore, the inversion is rather insensitive to large 
photospheric velocities, which rather are adjusted to reproduce the fore-mentioned velocity gradient.

Our observations do not allow for a detailed interpretation of the horizontal component of the magnetic field inside the umbra: the umbral Stokes $Q$ and $U$ signals are too weak to allow for robust results. Therefore we only comment on the longitudinal component. The overall magnetic field configuration remains rather constant inside the umbra during the flash, but we find subarcsecond fluctuations that could be produced by the flashes.

The situation is slightly different in the penumbra, though, perhaps because the field is more inclined (resulting in a stronger component perpendicular to the l.o.s.) or because it is weaker (or a combination of both reasons). We inverted a 2D subfield along the superpenumbra where running penumbral waves were clearly observed. The magnetic field vector inferred by the inversions is almost vertical at the boundary of the umbra, becoming more horizontal outwards. Further analysis of the entire time series have allowed us to measure the imprint of these waves in the magnetic field strength, which seems to be modulated with a peak-to-peak amplitude some $200 \mathrm{G}$. Our results, however, do not show a similar fluctuation of the magnetic field inclination, which suggests that the wavefront is able to change the field strength but not its geometry.

We find a trend of decreasing power at high temporal frequencies for more horizontal magnetic fields, for the l.o.s. velocity and magnetic field strength. In the outer penumbra we find an absence of high frequency power while there is increasingly more power at high frequencies towards the umbra. This observation is interesting in the context of wave propagation along inclined magnetic fields. Low frequency waves ( $p$-modes), that are normally evanescent in the low density chromosphere, can leak into the chromosphere along inclined magnetic fields (Suematsu 1990; De Pontieu et al. 2004). This mechanism has been identified as the driving mechanism of dynamic fibrils (Hansteen et al. 2006; De Pontieu et al. 2007; Heggland et al. 2007). This mechanism might well be at play for UFs and running penumbral waves too and our analysis demonstrates that the kind of observations we present here are well suited to study this in more detail. We leave the extension of the inversions to a wider FOV and inclusion of a wider range of inclination angles that are needed to be more conclusive on this matter to future work.

Acknowledgements. The Swedish 1-m Solar Telescope was operated by the Institute for Solar Physics of the Royal Swedish Academy of Sciences in the Spanish Observatorio del Roque de los Muchachos of the Instituto de Astrofísica de Canarias. We thank Eamon Scullion for valuable help during the observations. Part of the computations were performed on resources at Chalmers Centre for Computational Science and Engineering (C3SE) provided by the Swedish National Infrastructure for Computing (SNIC), with project number SNIC002-12-27.

\section{References}

Asensio Ramos, A. 2011, ApJ, 731, 27

Auer, L. 2003, in Stellar Atmosphere Modeling, eds. I. Hubeny, D. Mihalas, \& K. Werner, ASP Conf. Ser., 288, 3

Bard, S., \& Carlsson, M. 2010, ApJ, 722, 888

Barklem, P. S., \& O'Mara, B. J. 1998, MNRAS, 300, 863

Beck, C., Rezaei, R., \& Puschmann, K. G. 2013, A\&A, 553, A73

Beckers, J. M., \& Tallant, P. E. 1969, Sol. Phys., 7, 351

Bharti, L., Hirzberger, J., \& Solanki, S. K. 2013, A\&A, 552, L1

Bloomfield, D. S., Lagg, A., \& Solanki, S. K. 2007, ApJ, 671, 1005
Brault, J. W., \& Neckel, H. 1987, Spectral Atlas of Solar Absolute Disk-averaged and Disk-Center Intensity from 3290 to $12510 \AA$, ftp: //ftp.hs.uni-hamburg.de/pub/outgolng/FTS-Atlas

Bray, R. J., \& Loughhead, R. E. 1974, The solar chromosphere, Int. Astrophys. Ser. (London: Chapman and Hall)

Carlin, E. S., Asensio Ramos, A., \& Trujillo Bueno, J. 2013, ApJ, 764, 40

Cauzzi, G., Reardon, K. P., Uitenbroek, H., et al. 2008, A\&A, 480, 515

Centeno, R., Socas-Navarro, H., Collados, M., \& Trujillo Bueno, J. 2005, ApJ, 635,670

Collados, M., Rodríguez Hidalgo, I., Bellot Rubio, L., Ruiz Cobo, B., \& Soltau, D. 1999, in Astronomische Gesellschaft Meeting Abstracts, 13

de la Cruz Rodriguez, J. 2010, Ph.D. Thesis, Stockholm University, http://urn.kb.se/resolve?urn=urn:nbn: se: su:diva-43646

de la Cruz Rodríguez, J., Kiselman, D., \& Carlsson, M. 2011, A\&A, 528, A113

de la Cruz Rodríguez, J., Socas-Navarro, H., Carlsson, M., \& Leenaarts, J. 2012, A\&A, 543, A34

de la Cruz Rodríguez, J., \& Piskunov, N. 2013, ApJ, 764, 33

De Pontieu, B., Erdélyi, R., \& James, S. P. 2004, Nature, 430, 536

De Pontieu, B., Hansteen, V. H., Rouppe van der Voort, L., van Noort, M., \& Carlsson, M. 2007, ApJ, 655, 624

Felipe, T., Khomenko, E., \& Collados, M. 2010, ApJ, 719, 357

Felipe, T., Khomenko, E., \& Collados, M. 2011, ApJ, 735, 65

Gingerich, O., Noyes, R. W., Kalkofen, W., \& Cuny, Y. 1971, Sol. Phys., 18, 347

Hansteen, V. H., De Pontieu, B., Rouppe van der Voort, L., van Noort, M., \& Carlsson, M. 2006, ApJ, 647, L73

Heggland, L., De Pontieu, B., \& Hansteen, V. H. 2007, ApJ, 666, 1277

Henriques, V. M. J. 2012, A\&A, 548, A114

Henriques, V. M. J. 2013, Ph.D. Thesis, Stockholm University,

http://urn.kb.se/resolve?urn=urn: nbn: se : su: diva- 86798

Katsukawa, Y., Berger, T. E., Ichimoto, K., et al. 2007, Science, 318, 1594

Kneer, F., Mattig, W., \& Uexkuell, M. V. 1981, A\&A, 102, 147

Kosugi, T., Matsuzaki, K., Sakao, T., et al. 2007, Sol. Phys., 243, 3

Landi Degl'Innocenti, E., \& Landolfi, M. 2004, Polarization in Spectral Lines, Astrophys. Space Sci. Lib., 307

Leenaarts, J., Carlsson, M., Hansteen, V., \& Rouppe van der Voort, L. 2009, ApJ, 694, L128

López Ariste, A., Socas-Navarro, H., \& Molodij, G. 2001, ApJ, 552, 871

Manso Sainz, R., \& Trujillo Bueno, J. 2010, ApJ, 722, 1416

Press, W. H., Teukolsky, S. A., Vetterling, W. T., \& Flannery, B. P. 2002, Numerical recipes in $\mathrm{C}++$ : The art of scientific computing, 2nd edn (Cambridge University Press)

Rayrole, J. 1967, Ann. Astrophys., 30, 257

Rouppe van der Voort, L. H. M., Rutten, R. J., Sütterlin, P., Sloover, P. J., \& Krijger, J. M. 2003, A\&A, 403, 277

Ruiz Cobo, B., \& del Toro Iniesta, J. C. 1992, ApJ, 398, 375

Scharmer, G. B. 2006, A\&A, 447, 1111

Scharmer, G. B., Bjelksjo, K., Korhonen, T. K., Lindberg, B., \& Petterson, B. 2003, in SPIE Conf. Ser. 4853, eds. S. L. Keil, \& S. V. Avakyan, 341

Scharmer, G. B., Narayan, G., Hillberg, T., et al. 2008, ApJ, 689, L69

Scharmer, G. B., de la Cruz Rodríguez, J., Sütterlin, P., \& Henriques, V. M. J. 2013, A\&A, 553, A63

Schnerr, R. S., de La Cruz Rodríguez, J., \& van Noort, M. 2011, A\&A, 534, A45

Selbing, J. 2010, MSc Thesis, Stockholm University [arXiv: 1010.4142]

Shine, R. A., Title, A. M., Tarbell, T. D., et al. 1994, ApJ, 430, 413

Socas-Navarro, H. 2011, A\&A, 529, A37

Socas-Navarro, H., \& Trujillo Bueno, J. 1997, ApJ, 490, 383

Socas-Navarro, H., Trujillo Bueno, J., \& Ruiz Cobo, B. 2000a, ApJ, 544, 1141

Socas-Navarro, H., Trujillo Bueno, J., \& Ruiz Cobo, B. 2000b, Science, 288, 1396

Socas-Navarro, H., Trujillo Bueno, J., \& Ruiz Cobo, B. 2000c, ApJ, 530, 977

Socas-Navarro, H., Trujillo Bueno, J., \& Ruiz Cobo, B. 2001, ApJ, 550, 1102

Socas-Navarro, H., McIntosh, S. W., Centeno, R., de Wijn, A. G., \& Lites, B. W. 2009, ApJ, 696, 1683

Suematsu, Y. 1990, in Progress of Seismology of the Sun and Stars, eds. Y. Osaki \& H. Shibahashi (Berlin: Springer Verlag), Lect. Notes Phys., 367, 211 Thomas, J. H. 1984, A\&A, 135, 188

Trujillo Bueno, J., \& Landi Degl'Innocenti, E. 1996, Sol. Phys., 164, 135

Tsiropoula, G., Alissandrakis, C. E., \& Mein, P. 2000, A\&A, 355, 375

Tsuneta, S., Ichimoto, K., Katsukawa, Y., et al. 2008, Sol. Phys., 249, 167

van Noort, M. J., \& Rouppe van der Voort, L. H. M. 2008, A\&A, 489, 429

van Noort, M., Rouppe van der Voort, L., \& Löfdahl, M. G. 2005, Sol. Phys., 228,191

Vissers, G., \& Rouppe van der Voort, L. 2012, ApJ, 750, 22

Wittmann, A. 1969, Sol. Phys., 7, 366

Pages 12 to 15 are available in the electronic edition of the journal at http://www . aanda.org 


\section{Appendix A: The flat-field correction}

Especial care must be taken with the flat-fielding procedure. At the SST, the flat-field data are acquired while the telescope moves in circles around solar disk center, accumulating $\approx 1000$ images per wavelength and polarization state. Effectively, the data represent a temporal and spatial average of quiet-Sun data at solar disk center. Therefore, it is reasonable to assume that the observed line profile at each pixel should be almost identical, except for the dirt, wavelength dependent fringes and the prefilter transmission, that can change across the FOV.

The telecentric configuration used in CRISP is optimal to achieve high spatial-resolution observations, although the instrumental transmission profile is not constant across the field-ofview (FOV). Pixel-to-pixel wavelength shifts (cavity errors) are present due to imperfections in the surface of the etalons that cannot be infinitely flat. Additionally, imperfections in the coating of the etalons produce reflectivity variations across the FOV that change the width of the transmission profile (reflectivity errors).

These effects become obvious when a spectral line is present at the observed wavelength, producing intensity fluctuations across the FOV that correlate tightly with the cavity errors and, less obviously, with variations in the reflectivity.

While these instrumental effects are permanently anchored to the same pixels on the camera, solar features are moved around by differential seeing motions, however, the MOMFBD code is only able to process data where the intensity at any given pixel, is strictly changing due to atmospheric distortion. To overcome this problem Schnerr et al. (2011) proposed a strategy to characterize CRISP and prepare flat-field images that allow to reconstruct the data.

Their idea is founded on the assumption that each pixel observed the same quiet-Sun profile during the flat-field data acquisition. To remove the imprint of the quiet-Sun profile from each pixel, an iterative self-consistent scheme is used to separate the different contributions to the intensity profile at each pixel, which we summarize in the following lines. To reproduce the observed profile on a pixel-to-pixel basis, they assume that the intensity at each pixel can be expressed as,

$I_{\mathrm{obs}}(\lambda)=c_{0}\left(I_{\mathrm{QS}}(\lambda) * T\left(e_{\mathrm{ch}}, e_{\mathrm{rh}}\right)\right)\left(1+c_{3} \lambda\right)$,

where $I_{\mathrm{obs}}$ is the observed profile at a given pixel, $\lambda$ is the wavelength, $I_{\mathrm{QS}}$ is the estimate of the spatially-averaged quiet-Sun profile, $T$ is a theoretical CRISP transmission profile, $c_{0}$ is a multiplicative factor that scales the entire profile and $c_{3}$ accounts for shifts of the prefilter. $e_{\mathrm{ch}}$ and $e_{\mathrm{rh}}$ represent the high resolution etalon cavity error and reflectivity error respectively. The * stands for a convolution operator.

We simplify their strategy, by assuming constant reflectivities across the FOV. Neglecting the effect of reflectivity errors works particularly well in broad-lines like the $\lambda 8542$, given that the transmission profile is relatively narrow compared to the Doppler width of the line. It also reduces the time required to compute the fits, from hours to minutes on a single CPU, as no convolutions are required any longer. We also allowed to include two more terms to the prefilter polynomial, given that at this wavelength there are fringes that change as a function of wavelength, introducing some distortion in the profiles.

Figure A.1 (lower panel) illustrates a density map, where the profiles from all pixels have been divided by $c_{0}\left(1+c_{3} \lambda+c_{4} \lambda^{2}+\right.$ $c_{5} \lambda^{3}$ ) and shifted to the correct wavelength. The best estimate of the quiet-sun profile is plotted with a blue line. A tight correlation means that our model is able to reproduce the observed
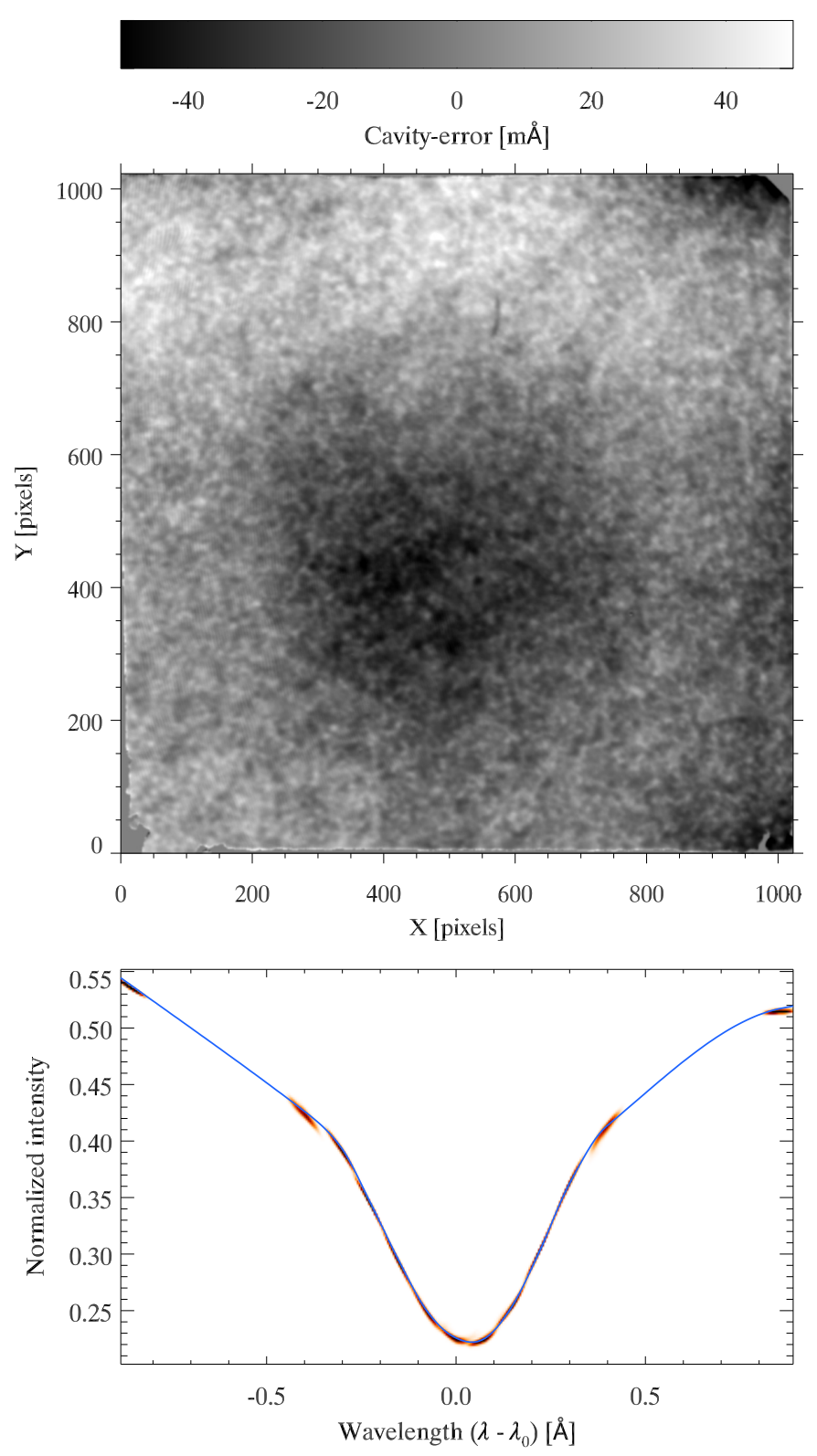

Fig. A.1. Top: map of the FPI cavity errors in units of wavelength shift. Bottom: density plot of all pixels in the summed flat-field data (orange clouds) that have been shifted to the correct wavelength and corrected for the different contributions in the model. The blue line is the average line profile described by a spline curve and closely resembles a disk center quiet Sun atlas profile.

profiles, despite the simplifications applied to the original model by Schnerr et al. (2011). A cavity-error map is also shown in Fig. A.1

\section{A.1. The CCD backscatter problem}

The back-illuminated Sarnoff CAM1M100 CCD cameras used in the CRISP instrument allow to acquire 35 frames-per-second, freezing atmospheric motions during each acquisition. These cameras work particularly well in visible light, but their efficiency decreases steeply in the infrared.

Above approximately $7000 \AA$, the CCD becomes semitransparent and the images show a circuit pattern that is not present at other wavelengths. de la Cruz Rodriguez (2010) 

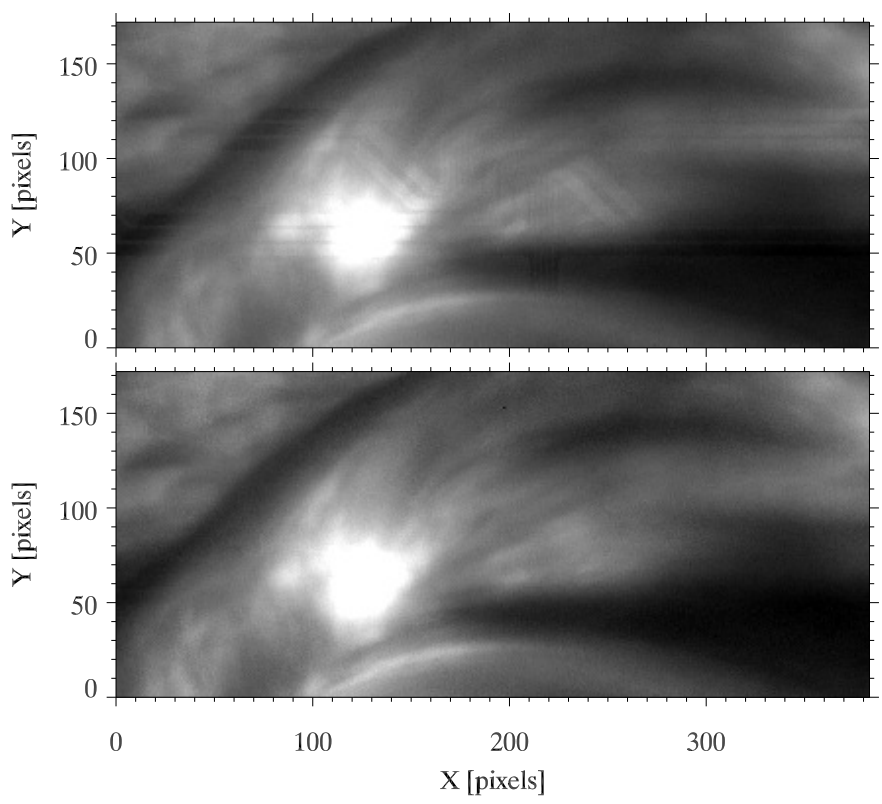

Fig. A.2. Top: subfield from an individual acquisition, where traditional dark-current and flat-field corrections have been applied. An electronic circuit pattern from the CCD chip is visible in the background. Bottom: same subfield, backscatter corrected.

explains a way to overcome this issue and properly apply the flat-field correction to the data. Furthermore, the images show a circuit-like pattern that cannot be removed by traditional darkfield and flat-field corrections, as illustrated in Fig. A.2 (top panel). Examination of pinhole data and flat-fielded data shows that:

1. there is a diffuse additive stray-light contribution in the whole image;

2. the stray-light contribution is much smaller in the circuit pattern and the gain appears to be enhanced.

These problems can be explained by a semi-transparent CCD with a diffusive medium behind it, combined with an electronic circuit located right behind the CCD that is partially reflecting and therefore also less transparent to the scattered light. Under normal conditions an image recorded with a $\mathrm{CCD}, I_{\mathrm{o}}$, can be described in terms of the dark-field $(D)$, the gain factor $\left(G_{\mathrm{f}}\right)$ and the real image $\left(I_{\mathrm{r}}\right)$ :

$I_{\mathrm{o}}=D+\left(G_{\mathrm{f}} I_{\mathrm{r}}\right)$

However, in the infrared we need a more complicated model:

$I_{\mathrm{o}}=D+f(1-f)\left[\left(G_{\mathrm{b}} G_{\mathrm{f}} I_{\mathrm{r}}\right) * P\right] G_{\mathrm{b}}+f G_{\mathrm{f}} I_{\mathrm{r}}$

where $f$ represents the overall fraction of light absorbed by the $\mathrm{CCD}, G_{\mathrm{b}}$ is the gain for light illuminating the CCD from the back which should account for the electronic circuit pattern. In the following, we refer to $G_{\mathrm{b}}$ as backgain. $P$ is a point spread function (PSF) that describes the scattering properties of the dispersive screen. In the backscatter term we assume that $(1-f) G_{\mathrm{f}} I_{\mathrm{r}} G_{\mathrm{b}}$ is transmitted to the diffusive medium where it is scattered. A fraction of the scattered light returns to the CCD, passing again through the circuit. The sketch in Fig. A.3 shows a schematic representation of the structure of the camera and the path followed by the light beam.

To obtain the real intensity $I_{\mathrm{r}}$, the PSF $P$, the back-gain $G_{\mathrm{b}}$ and the front-gain $G_{\mathrm{f}}$ must be known. The transparency factor is

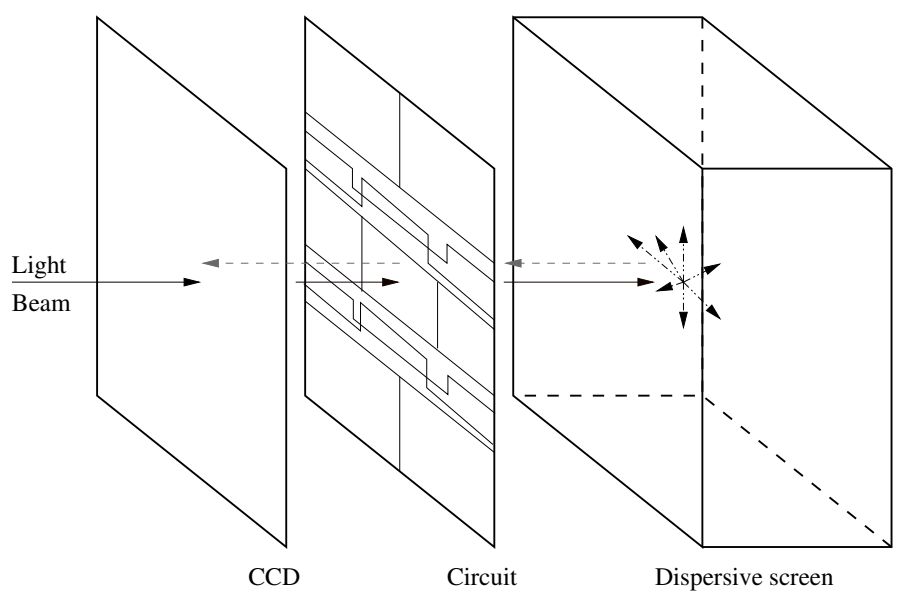

Fig. A.3. Sketch showing a conceptual model of the camera. The dark arrows indicate the incoming light beam from the telescope and the gray arrows represent the back-scattered light that returns to the CCD.

assumed to be smooth because the properties of the dispersive screen seem to be homogeneous across the FOV. This allows us to include $f$ in the front and the back gain factors

$$
\begin{aligned}
G_{\mathrm{b}^{\prime}} & =\sqrt{1-f} G_{\mathrm{b}} \\
G_{\mathrm{f}^{\prime}} & =f G_{\mathrm{f}},
\end{aligned}
$$

so Eq. (A.3) becomes:

$I_{\mathrm{o}}=D+G_{\mathrm{b}^{\prime}}\left[\left(G_{\mathrm{b}^{\prime}} G_{\mathrm{f}^{\prime}} I_{\mathrm{r}}\right) * P\right]+G_{\mathrm{f}^{\prime}} I_{\mathrm{r}}$.

This problem is linear and invertible, but a direct inversion would be expensive, given the dimensions of the problem. A numerical approach can be used to iteratively solve the problem. We define

$\hat{J}=\left(G_{\mathrm{b}^{\prime}} G_{\mathrm{f}^{\prime}} I_{\mathrm{r}}\right) * P$.

The back gain $G_{\mathrm{b}^{\prime}}$ is assumed to be 1 for every pixel in the first iteration. Furthermore, we assume that the product $G_{\mathrm{f}^{\prime}} I_{\mathrm{r}}$ can be estimated from Eq. (A.2) ignoring back scattering, i.e., $G_{\mathrm{f}^{\prime}} I_{\mathrm{r}} \approx$ $I_{\mathrm{O}}-D$. These values are of the same order of magnitude as the final solution, and therefore correspond to a reasonable choice of initialization:

$\hat{J}_{0} \approx\left\langle G_{\mathrm{b}^{\prime}} G_{\mathrm{f}^{\prime}} I_{\mathrm{r}}\right\rangle \approx\left\langle G_{\mathrm{b}^{\prime}}\left(I_{\mathrm{o}}-D\right)\right\rangle$.

We assume rotational symmetry to construct the 2D PSF $P$, which is parameterized by placing node points along the radial component. The latter are connected by straight lines in logarithmic scale. To estimate the initial guess of the node points we use an angular average obtained from a pinhole image. The small diameter of the pinhole only allows to estimate accurately the central part of the PSF. The wings of our initial guess are extrapolated using a power-law. The estimate of $G_{f^{\prime}} I_{\mathrm{r}}$ is then

$G_{\mathrm{f}^{\prime}} I_{\mathrm{r}}=I_{\mathrm{o}}-D-G_{\mathrm{b}^{\prime}} \hat{J}$

which can be used to compute a new estimate $\hat{J}$. This new value of $\hat{J}$ is used to improve our estimate of $G_{\mathrm{b}^{\prime}}$, by applying the same procedure to images that contain parts being physically masked $\left(I_{\mathrm{r}}=0\right)$, as in Fig. A.4. In the masked parts where $I_{\mathrm{r}} \equiv 0$ we have,

$I_{\mathrm{o}}-D-G_{\mathrm{b}^{\prime}} \hat{J}=0$, 


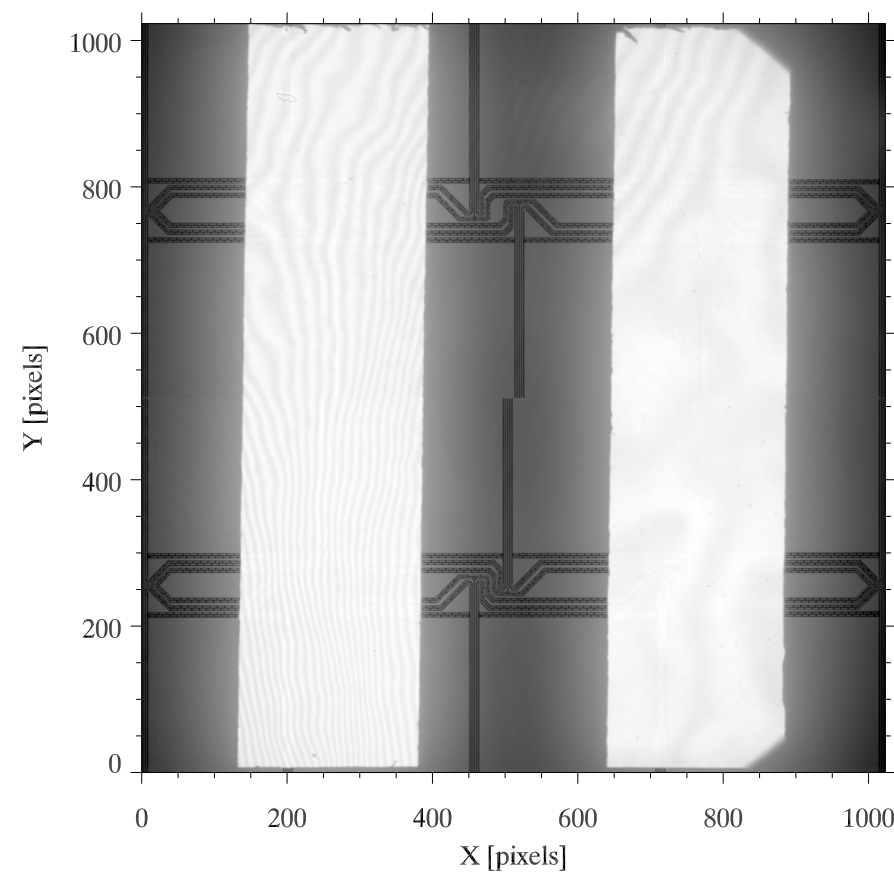

Fig. A.4. Calibration data acquired to infer the backgain and the PSF describing the backscatter problem. The dark areas are physically masked on the focal plane of the telescope. The color scale is saturated to enhance the CCD chip pattern.

so the back-gain can be computed directly:

$G_{\mathrm{b}^{\prime}}=\frac{I_{\mathrm{o}}-D}{\hat{J}}$

Thus, every pixel must have been covered by the mask at least once in a calibration image in order to allow the calculation of the back-gain. With the new $G_{\mathrm{b}^{\prime}}$ we can recompute a new estimate of $G_{f^{\prime}} I_{\mathrm{r}}$. This procedure, enclosing Eqs. (A.7)-(A.9) is iterated, until $G_{\mathrm{f}^{\prime}} I_{\mathrm{r}}$ and $I_{\mathrm{o}}$ are consistent.

We now need to specify a measure that describes how well the data are fitted by the estimate of $P$ and $G_{\mathrm{b}^{\prime}}(P)$. Since both $G_{f^{\prime}} I_{\mathrm{r}}$ and $G_{\mathrm{b}^{\prime}}$ are computed based on self-consistency, we iteratively need to fit only the parameters of the PSF. We assume that the PSF is circular-symmetric and apply corrections to the PSF at node points placed along the radius.

We use Brent's Method described by Press et al. (2002) to minimize our fitness function. This algorithm does not require the computation of derivatives with respect to the free parameters of the problem. When the opaque bars block a region of the CCD, an estimate of the back gain can be calculated for a given PSF according to Eq. (A.9). In our calibration data, the four bars of width $L$ are displaced $0.5 L$ from one image to the next. This overlapping provides two different measurements of the back gain on each region of the CCD. However, as the location of the bars changes on each image, the scattered light contribution is different for each of these measurements of the back gain. Our fitness function minimizes the difference between these two measurements of the back gain. Note that two nested iterative loops are needed to compute the parameters of the PSF. The inner loop is used to compute a self-consistent value of $G_{\mathrm{f}^{\prime}} I_{\mathrm{r}}$ for any given PSF $P$, whereas in the outer loop the parameters of a guessed PSF are fitted to compensate for the backscatter problem. We have summarized the work flow in Fig. A.5.

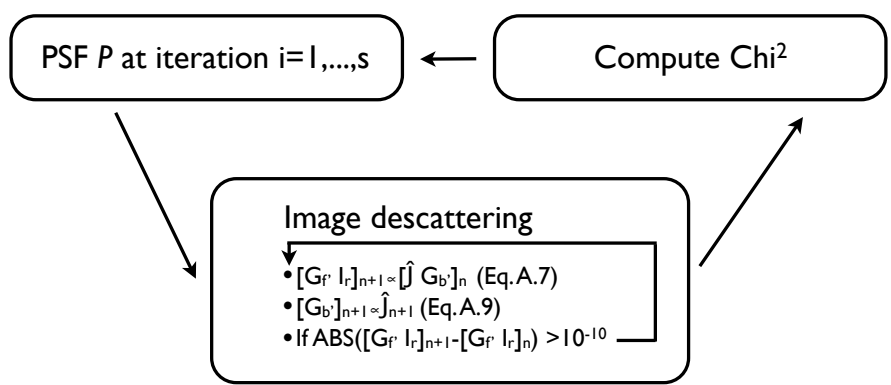

Fig. A.5. Work flow of out iterative scheme to fit the parameters of the backscatter PSF. The scheme has two nested iterative schemes, the inner loop is used to obtain the descattered image $\left(G_{\mathrm{f}^{\prime}} I_{\mathrm{r}}\right)$ for each set of guessed PSF parameters (the outer loop).

Having thus obtained the PSF $P$ and the back-gain $G_{\mathrm{b}^{\prime}}$, we obtain $G_{\mathrm{f}^{\prime}}$ by recording conventional flats and assuming $I_{\mathrm{r}}$ is a constant in order to obtain $G_{f^{\prime}}$ from Eq. (A.4).

\section{Appendix B: The SST polarization model at $1854 \mathrm{~nm}$}

The optical path of the SST, from the $1 \mathrm{~m}$ lens down to the cameras, contains optical elements that can polarize light. Therefore, polarimetric observations at the SST must be calibrated.

At the SST this calibration is split in two parts: the optical table and the turret. The former is calibrated daily, given that the observer can easily place a linear polarizer and a retarder to generate known polarization states, and analyze how the optical table changes the polarization (see van Noort \& Rouppe van der Voort 2008, for further details).

The result is a modulation matrix $\mathbf{M}_{\text {table }}$ that allows to convert the observed polarization states $\boldsymbol{I}_{\mathrm{lc}} \equiv\left(I_{l c 0}, I_{l c 1}, I_{l c 2}, I_{l c 3}\right)^{\mathrm{T}}$ into Stokes parameters $\boldsymbol{I} \equiv(I, Q, U, V)^{\mathrm{T}}$ parameters, using a linear combination:

$\boldsymbol{I}^{\prime}=\mathbf{M}_{\mathrm{table}}^{-1} \cdot \boldsymbol{I}_{\mathrm{lc}}$,

where the prime symbol indicates that only the optical table has been calibrated.

In principle, if the telescope was built with non-polarizing elements then $\left(I^{\prime}, Q^{\prime}, U^{\prime}, V^{\prime}\right) \equiv(I, Q, U, V)$, but this is not the case. The turret has mirrors and a lens that change the polarization properties of the incoming light. In fact, the polarizing properties of the turret change with the pointing. Therefore, a final transformation $\left(\mathbf{M}_{\mathrm{tel}}\right)$ is needed to obtain telescope-polarization free Stokes parameters:

$\boldsymbol{I}=\mathbf{M}_{\mathrm{tel}}^{-1} \cdot \boldsymbol{I}^{\prime} \equiv \mathbf{M}_{\mathrm{tel}}^{-1} \cdot\left(\mathbf{M}_{\mathrm{table}}^{-1} \cdot \boldsymbol{I}_{\mathrm{lc}}\right)$

Selbing (2010) studied the polarizing properties of the SST and proposed a model to characterize the telescope induced polarization at $630 \mathrm{~nm}$. In this work, we use a similar model. Their work allows to compute $\mathbf{M}_{\text {tel }}$ based on the azimuth and elevation parameters of the telescope pointing.

Calibration images have been recorded during an entire day, using a $1-\mathrm{m}$ polarizer mounted at the entrance lens of the SST. The polarizer rotates $360 \mathrm{deg}$ in steps of $5 \mathrm{deg}$ and several frames are acquired for each angle. These data are used to derive the parameters of the model proposed by Selbing (2010) at $854 \mathrm{~nm}$. This model is already presented in de la Cruz Rodriguez (2010) and included here for completeness.

Each polarizing optical element of the telescope is represented with a Mueller matrix. $\mathbf{L}$ represents the entrance lens. 
The form of the lens matrix represents a composite of random retarders, so it demodulates the light without polarizing it. The reference for $Q$ is aligned with the $1 \mathrm{~m}$ linear polarizer axis and the values of the matrix are measured in that frame:

$\mathbf{L}\left(l_{0}, l_{1}, l_{2}, l_{3}, l_{4}\right)=\left(\begin{array}{cccc}1 & 0 & 0 & 0 \\ 0 & l_{0} & l_{1} & -l_{2} \\ 0 & l_{1} & l_{3} & l_{4} \\ 0 & l_{2} & -l_{4} & \left(l_{0}+l_{3}-1\right)\end{array}\right)$.

Mirrors are noted with $\mathbf{M}$ and have two free parameters. Assuming a wave that propagates along the $z$-axis and oscillates in the $x-y$ plane, the parameters are the de-attenuation term $R$ between the $x y$ components of the electromagnetic wave and the phase retardance $\delta$ produced by the mirror. This form of $\mathbf{M}$ assumes that $Q$ is perpendicular to the plane of incidence:

$\mathbf{M}(R, \delta)=\left(\begin{array}{cccc}\frac{1}{2}+\frac{1}{2} R & \frac{1}{2}-\frac{1}{2} R & 0 & 0 \\ \frac{1}{2}-\frac{1}{2} R & \frac{1}{2}+\frac{1}{2} R & 0 & 0 \\ 0 & 0 & -\sqrt{R} \cos \left(\frac{\pi}{180} \delta\right) & -\sqrt{R} \sin \left(\frac{\pi}{180} \delta\right) \\ 0 & 0 & \sqrt{R} \sin \left(\frac{\pi}{180} \delta\right) & -\sqrt{R} \cos \left(\frac{\pi}{180} \delta\right)\end{array}\right)$.

Finally, $\mathbf{R}(\alpha)$ corresponds to a rotation to a new coordinate frame:

$\mathbf{R}(\alpha)=\left(\begin{array}{cccc}1 & 0 & 0 & 0 \\ 0 & \cos \left(\frac{\pi}{90} \alpha\right) & \sin \left(\frac{\pi}{90} \alpha\right) & 0 \\ 0 & -\sin \left(\frac{\pi}{90} \alpha\right) & \cos \left(\frac{\pi}{90} \alpha\right) & 0 \\ 0 & 0 & 0 & 1\end{array}\right)$

Selbing (2010) builds the telescope model using the Mueller matrix of each polarizing element, as a function of the azimuth $(\varphi)$ and elevation $(\theta)$ angles of the Sun at the time of the observation (Eq. (B.3)). We have included the conversion factor from degrees to radians in the matrices, thus all the angles are given in degrees:

$$
\begin{aligned}
M_{\mathrm{tel}}(\theta, \varphi)= & R_{\mathrm{f}+}\left(c_{11}\right) M_{\mathrm{s}}\left(c_{9}, c_{10}\right) \cdot M_{\mathrm{f}}\left(c_{7}, c_{8}\right) \cdot \\
& \cdot R_{\mathrm{f}-}\left(c_{11}\right) \cdot R_{\mathrm{az}}(\varphi) \cdot M_{\mathrm{az}}\left(c_{5}, c_{6}\right) \cdot R_{\mathrm{el}}(\theta) \\
& \cdot M_{\mathrm{el}}\left(c_{5}, c_{6}\right) \cdot L\left(c_{0}, c_{1}, c_{2}, c_{3}, c_{4}\right),
\end{aligned}
$$

Table B.1. Parameters of the telescope model obtained from calibration data at $8542 \mathrm{~nm}$.

\begin{tabular}{c|l}
\hline \hline Parameter & Fitted value \\
\hline$c_{0}$ & $+9.596161 \times 10^{-1}$ \\
$c_{1}$ & $-2.541791 \times 10^{-3}$ \\
$c_{2}$ & $-8.337375 \times 10^{-3}$ \\
$c_{3}$ & $+9.640901 \times 10^{-1}$ \\
$c_{4}$ & $-1.875988 \times 10^{-2}$ \\
$c_{5}$ & $+9.164327 \times 10^{-1}$ \\
$c_{6}$ & $+1.465364 \times 10^{1}$ \\
$c_{7}$ & $+1.017471 \times 10^{0}$ \\
$c_{8}$ & $+1.326977 \times 10^{0}$ \\
$c_{9}$ & $+1.010035 \times 10^{0}$ \\
$c_{10}$ & $-1.592178 \times 10^{0}$ \\
$c_{11}$ & $+4.000000 \times 10^{0}$ \\
\hline
\end{tabular}

where $c_{1,2, \ldots, 11}$ are the parameters of the model, computed using a least-squares fit to the observed data. The fitted values are summarized in Table B.1.

Note that the polarizer has a significant leak of unpolarized light at $854.2 \mathrm{~nm}$. Using small samples of the sheets used to construct the $1-\mathrm{m}$ polarizer, we have estimated the extinction ratio of the $1-\mathrm{m}$ polarizer to be approximately $40 \%$, so the parameters of the lens have been determined assuming that value.

The linear polarization reference is defined by the first mirror after the lens, however this is not very useful in practice because the turret introduces image rotation along the day. Instead, we use the solar north as a reference for positive $Q$ by applying an extra rotation to $\mathbf{M}_{\text {tel }}$.

The full-Stokes Ca II images in Fig. 2 have been calibrated using the telescope model described in this section. 